\title{
A multi-omic analysis reveals the role of fumarate in regulating the virulence of enterohemorrhagic Escherichia coli
}

\author{
Cheng-Ju Kuo ${ }^{1,2}$, Sin-Tian Wang ${ }^{2}$, Chia-Mei Lin', Hao-Chieh Chiu ${ }^{3}$, Cheng-Rung Huang ${ }^{1,2}$, Der-Yen Lee ${ }^{4,5}$, \\ Geen-Dong Chang ${ }^{5}$, Ting-Chen Chou', Jenn-Wei Chen $\mathbb{1}^{2,6}$ and Chang-Shi Chen $\mathbb{1}^{1,2}$
}

\begin{abstract}
The enteric pathogen enterohemorrhagic Escherichia coli (EHEC) is responsible for outbreaks of bloody diarrhea and hemolytic uremic syndrome (HUS) worldwide. Several molecular mechanisms have been described for the pathogenicity of EHEC; however, the role of bacterial metabolism in the virulence of EHEC during infection in vivo remains unclear. Here we show that aerobic metabolism plays an important role in the regulation of EHEC virulence in Caenorhabditis elegans. Our functional genomic analyses showed that disruption of the genes encoding the succinate dehydrogenase complex (Sdh) of EHEC, including the sdhA gene, attenuated its toxicity toward C. elegans animals. Sdh converts succinate to fumarate and links the tricarboxylic acid (TCA) cycle and the electron transport chain (ETC) simultaneously. Succinate accumulation and fumarate depletion in the EHEC sdhA mutant cells were also demonstrated to be concomitant by metabolomic analyses. Moreover, fumarate replenishment to the sdhA mutant significantly increased its virulence toward C. elegans. These results suggest that the TCA cycle, ETC, and alteration in metabolome all account for the attenuated toxicity of the sdhA mutant, and Sdh catabolite fumarate in particular plays a critical role in the regulation of EHEC virulence. In addition, we identified the tryptophanase (TnaA) as a downstream virulence determinant of SdhA using a label-free proteomic method. We demonstrated that expression of thaA is regulated by fumarate in EHEC. Taken together, our multi-omic analyses demonstrate that sdhA is required for the virulence of EHEC, and aerobic metabolism plays important roles in the pathogenicity of EHEC infection in C. elegans. Moreover, our study highlights the potential targeting of SdhA, if druggable, as alternative preventive or therapeutic strategies by which to combat EHEC infection.
\end{abstract}

\section{Introduction}

Enterohemorrhagic Escherichia coli (EHEC) is one of the Shiga-like toxin (Stx) producing pathogens, and can cause severe diarrhea, hemorrhagic colitis, and hemolytic uremic syndrome (HUS) in humans ${ }^{1}$. Besides the Stxs in

\footnotetext{
Correspondence: Jenn-Wei Chen (jc923@mail.ncku.edu.tw) or Chang-Shi Chen (cschen@mail.ncku.edu.tw)

${ }^{1}$ Department of Biochemistry and Molecular Biology, College of Medicine, National Cheng Kung University, Tainan, Taiwan

${ }^{2}$ Institute of Basic Medical Sciences, College of Medicine, National Cheng Kung University, Tainan, Taiwan

Full list of author information is available at the end of the article

These authors contributed equally: Cheng-Ju Kuo, Sin-Tian Wang, Chia-Mei Lin.

Edited by A. Finazzi-Agrò.
}

EHEC, several other virulence determinants have been identified, including the locus of enterocyte effacement (LEE) and the F-like virulence plasmid $p O 157^{2}$. It has been reported EHEC can produce indoles, from tryptophan metabolism catalyzed by the tryptophanase TnaA, to paralyze and kill $C$. elegans animals ${ }^{3,4}$. Moreover, the secreted indoles produced by EHEC act as signal molecules to regulate LEE genes expression, and together with the $t n a A$ gene are required for the formation of attaching and effacing (A/E) lesions in cultured mammalian cells ${ }^{5}$. Aerobic respiration has also been implicated to be required for the colonization of EHEC in the mouse 
intestine $^{6}$. This report is in agreement with the notion that a zone adjacent to the GI tract mucosa, the potential in vivo microenvironment for EHEC, is aerobic caused by diffusion of oxygen from the capillary network at the tips of microvilli ${ }^{7}$. An in vitro study also showed that the expression profile of the LEE genes in EHEC can be regulated by the catabolite of glucose metabolism, fructose-1, 6-bisphsphate (FBP), in a catabolite repressor/ activator protein Cra-dependent manner ${ }^{8}$. These reports together suggest that carbon and central metabolic pathways might regulate the pathogenesis of EHEC in the localized microenvironment in vivo.

In order to adapt to local microenvironments, escape host defense, and causes disease during infection within the GI tract, EHEC can sense nutrients and chemical signals produced by either host or commensal microbiota ${ }^{9-12}$. EHEC also responds to lipid metabolites produced by host and microbiota in the ileum and colon, such as short-chain fatty acids (SCFAs) and ethanolamine (EA), to modulate the motility, adhesion, and virulence ${ }^{13-16}$. Moreover, the transportation and utilization of fucose, made available through the cleavage of host glycans by commensal microbiota, modulates EHEC pathogenicity in a FusKR two component system-dependent manner ${ }^{17}$. All these reports support the notion that nutrient metabolism and metabolites from host and microbiota work in concert to regulate EHEC virulence in vivo.

Owing to the lack of mouse models for studying EHEC infection $^{18}$, we and others applied the model organism $C$. elegans to study EHEC infection in vivo ${ }^{3,19}$. By screening an EHEC transposon mutagenesis library, we have identified potential bacterial virulence determinants involved in the pathogenicity of EHEC in C. elegans. Of note, we identified one transposon mutant of the $s d h A$ gene and one mutant of the $s d h C$ gene, these two genes encode the catalytic and ubiquinone interacting subunits respectively for the succinate dehydrogenase complex (Sdh). Sdh serves dual roles both in the TCA cycle (the sixth enzyme) and ETC (the complex II) in aerobic metabolism. However, the roles of bacterial central metabolism, including the TCA cycle and ETC, in regulating the virulence of EHEC during infection are still largely unknown. Herein, we further characterized how loss-of-function of the $s d h$ genes, particularly in the $s d h A$ mutants, can influence the virulence of EHEC, and how a Sdh catabolite, i.e., fumarate, could regulate the pathogenicity of EHEC in $C$. elegans.

\section{Materials and methods}

\section{Nematode strains and maintenance of $C$. elegans}

The nematode strains used in this study are listed in Supplemental Table S1. Worms were handled in the laboratory as described previously and were maintained on Nematode Growth Medium (NGM) agar plate fed with the standard and non-pathogenic laboratory $E$. coli strain OP50 as a normal food source ${ }^{20}$.

\section{Bacterial strains and plasmids}

The bacterial strains used in this study are listed in Supplemental Table S2. The enterohemorrhagic E. coli O157:H7 clinical isolates, including the EDL933 strain and the HER1266 strain, were from the Bioresource Collection and Research Center, Taiwan. All EHECrelated biohazardous wastes were disinfected and disposed according to the Biosafety Level 2 (BSL-2) regulation. All bacterial gene deletion strains were constructed by using the Lambda Red recombinase system $^{21,22}$ described in the supplemental information section. The genotypes/mutations of all bacterial strains used in the study were confirmed by DNA sequencing. The plasmids used in this study are listed in Supplemental Table S3. The ampicillin-resistant plasmid $p F P V 25.1$, which constitutively expressed green fluorescent protein (GFP) ${ }^{23}$, was transformed to OP50 and EDL933 strain to determine colonization status of live bacteria in the intestine of C. elegans. The $s d h C D A B$ operon expressing plasmid, $p W F 134$, was constructed by cloning the whole $s d h C D A B$ operon $(4769 \mathrm{bp})$ into the expression vector pQE30 (Qiagen) between the SphI and SalI restriction enzyme sites. The $s d h C D A B$ operon was amplified by PCR from EDL933 genomic DNA with the primers $5^{\prime}$-ACA TGC ATG CTT AAG GTC TCC TTA GCG CC-3' and 5'ACG CGT CGA CGC CGC ATC CGG CAC TGG TTG3'. The PCR amplified fragment was double digested with SphI and SalI restriction enzymes (NEB). The digested PCR product was extracted from agarose gels with QIAquick Gel Extraction Kit (Qiagen), and then was ligated with SphI/SalI-digested pQE30 vector to obtain the plasmid pWF134. Authentication of all the plasmids used in this study was reconfirmed by DNA sequencing. All the primers used in the study are list in Supplemental Table S4.

\section{Construction of the EHEC transposon mutagenesis library}

To generate the EDL933 genome-wide transposon mutants, we used the EZ-Tn5 $<$ R6Kyori/KAN-2 $>$ Tnp Transposome Kit (Epicentre). The EZ-Tn $5<\mathrm{R} 6 \mathrm{~K} \gamma$ ori/ KAN-2 > Transposon contains an R6K $\gamma$ conditional origin of replication (R6K $\gamma$ ori) and the Tn903 kanamycin resistance gene $\left(\operatorname{Kan}^{\mathrm{R}}\right)$ that is functional in E. coli, flanked by hyperactive 19 base pair Mosaic End (ME) EZ-Tn5 Transposase recognition sequences. The EZ-Tn5 system has allowed random mutagenesis in many bacterial species (www.epibio.com). To obtain EDL933 mutants, a 100 $\mu \mathrm{L}$ aliquot of the EDL933 electrocompetent cells was mixed with $1 \mu \mathrm{L}(25 \mathrm{ng} / \mu \mathrm{L})$ transposon DNA (EZ-Tn $5<$ R6Kyori/KAN-2 > Tnp Transposome Mutagenesis kit), incubated at $37^{\circ} \mathrm{C}$ overnight. The $\mathrm{Kan}^{\mathrm{R}}$ colonies were 
picked into the 96-well plates with LB broth contained 50 $\mu \mathrm{g} / \mathrm{mL}$ kanamycin, and the entire EDL933 transposome library was stored at $-80^{\circ} \mathrm{C}$.

\section{Screening of the EDL933 transposome library}

The detailed screening method is described and illustrated in the Supplemental Information (Figure S1). In brief, the L1 stage $C$. elegans glp-4(bn2) mutant animals, with germ-line proliferation defect developed at the restrictive temperature $\left(25^{\circ} \mathrm{C}\right)$, were seeded on an NGM plate with E. coli OP50 at Day 1. At the same time, the EDL 933 transposon mutagenesis library, stored in 96well plates, were replicated in LB broth containing $50 \mu \mathrm{g} /$ $\mathrm{mL}$ Kanamycin (Kan) and put in a $37^{\circ} \mathrm{C}$ incubator for 16-18 $\mathrm{h}$. On the next day (Day 2), the entire library was triplicated into 96-well plates containing LB broth with $50 \mu \mathrm{g} / \mathrm{mL}$ Kan and cultured at $37^{\circ} \mathrm{C}$ for another 16 to 18 h. At Day 3 until glp-4 (bn2) animals had reached to the L4 larva to young adult stage, the worms were washed by M9 buffer and added into the 96-well plates (15-20 worms/well) and incubated at $25^{\circ} \mathrm{C}$ with constant agitation at $70 \mathrm{rpm}$. After 8 days, the O.D.595 values of each well were detected by DTX 800 Multimode Detector (Beckman Coulter). The O.D. 595 value was close to 0.5 when worms were cultured with $E$. coli strain OP50 (as negative control). In contrast, the O.D.595 value was around 1.0 when the worms were fed with EHEC wildtype EDL933 (as positive control). The hits/candidates with a decreased pathogenic phenotype toward C. elegans were selected with the O.D. value that was significantly lower compared to the EHEC wild-type EDL933 positive controls $(P<0.05)$.

\section{Identification of the mutation sites of the selected Tn5 transposition clones}

The insertion site of any chosen EDL933 mutant clones was determined by the method described in the EZ-Tn5 Transposome Kit (Epicentre). In brief, the genomic DNA from the clone of interest was prepared by EasyPure Genomic DNA Spin kit (Bioman Scientific). Then, the genomic DNA was digested by EcoRV that cuts near the end of the EZ-Tn5 transposon. The resulted fragmented genomic DNA was self-ligated by T4 ligase. The ligation mixture was electroporated into the $E$. coli pir + electrocompetent cell and selected by Kan. Finally, the plasmids DNA of the rescued $\mathrm{Kan}^{\mathrm{R}}$ clones were sequenced using the forward and reverse EZ-Tn5 $<$ R6K $\gamma$ ori/KAN-2 $>$ Transposon-specific primers supplied in the kit system to identify the insertion site.

\section{EHEC killing assays}

C. elegans plate-based killing by E. coli O157:H7 strains were conducted as our previous published procedures $^{19,24}$. In brief, $30 \mu \mathrm{L}$ of the overnight $E$. coli LB broth culture was spread on $5.0 \mathrm{~cm}$ NGM agar plates and incubated overnight at $37^{\circ} \mathrm{C}$. On the next day, after equilibrating the plates to room temperature, about 50 synchronized late L4 to young adult stage C. elegans animals were transferred to each plate and kept at $20^{\circ} \mathrm{C}$. Animals were transferred to fresh plates daily during the progeny production period and monitored daily for dead animals. The experiment was performed independently three times with approximately 100 worms per E. coli strain each time at $20^{\circ} \mathrm{C}$. Survival analysis was performed using GraphPad Prism 6.0 (GraphPad Software, La Jolla, CA). The Mantel-Cox log-rank test was used to assess statistical significance of difference in survival, and $P$ values $<0.05$ were considered significant. For the succinate and fumarate treatment assays, bacteria were grown overnight at $37^{\circ} \mathrm{C}$ in LB broth containing $2.5 \mathrm{mM}$ succinic acid (Merck) or $2.5 \mathrm{mM}$ fumaric acid (Merck) and adjusted to $\mathrm{pH} 6.6$ by $\mathrm{NaOH}$, and spread on NGM agar plates also containing $2.5 \mathrm{mM}$ succinic acid or $2.5 \mathrm{mM}$ fumaric acid and adjusted to $\mathrm{pH} 6.2$ by $\mathrm{NaOH}$. For the anaerobic condition assay, NGM agar plates containing bacterial lawn $(100 \mu \mathrm{L}$ per plate $)$ were grown at $\mathrm{BBL}$ GasPak Anaerobic Systems at room temperature overnight. To ensure that worms were fed with bacteria from anaerobic culture continuously, worms were transferred to fresh prepared plates daily.

\section{Quantification of bacterial intestinal colonization}

Measurement of live bacterial number colonized in the intestine of $C$. elegans was performed as our previous published procedures ${ }^{19,24}$. In brief, N2 worms were fed with bacteria, including OP50, EDL933, EDL933: $\Delta s d h A$, or EDL933: $\Delta s d h A-p W F 134$, for 1 day at $20^{\circ} \mathrm{C}$ and then transferred to the OP50 bacterial plates for another three days at $20^{\circ} \mathrm{C}$. In order to keep the GFP-expressing pFPV25.1 plasmid, OP50-GFP, and EDL933-GFP were inoculated on the plates with ampicillin. On day 4, worms were washed out from the plate using M9 buffer treated with $25 \mathrm{mM}$ levamisole and collected by centrifugation at $1000 \times g$ for $1 \mathrm{~min}$. The infected worms were washed by M9 buffer containing $25 \mathrm{mM}$ levamisole for another 10 times. The infected worms then were incubated with M9 containing $25 \mathrm{mM}$ levamisole, 100 $\mathrm{mg} / \mathrm{mL}$ gentamicin and $1 \mathrm{mg} / \mathrm{mL}$ ampicillin for $1-2 \mathrm{~h}$ at room temperature to remove the bacteria outside of the animals. These antibiotics were eliminated by washing the worms in M9 buffer with $25 \mathrm{mM}$ levamisole three times. After the final wash, ten worms were picked randomly into $100 \mu \mathrm{L}$ M9 buffer in an Eppendorf microtube, pulverized for $1 \mathrm{~min}$ using a sterile plastic pestle, and plated on LB agar containing ampicillin after serial dilution. The number of bacterial cells (colony number) was determined and the colony-forming unit (CFU) per worm was calculated. Student's $t$-test was 
used to determine the significance of the differences $(P<0.05)$.

\section{Microvillar ACT-5 cellular localization}

Measurement of ectopic ACT-5 expression in the intestine of C. elegans was performed as described ${ }^{19,24}$. In brief, L4 larvae of the GK454 strain, the unc-119 mutant with dkIs247[act-5p::mCherry::HA::act-5, unc119(+)] transgene, were fed on bacterial lawns of OP50, EDL933, EDL933: $\Delta s d h A$, or EDL933: $\Delta s d h A-p W F 134$ for 4 days at $20^{\circ} \mathrm{C}$. The microvillar ACT-5 ectopic expression was determined by fluorescence microscopy.

\section{Metabolomic analysis}

Bacteria were cultured in LB broth at $37^{\circ} \mathrm{C}$ for $16-18 \mathrm{~h}$ with shaking at $220 \mathrm{rpm}$. Bacteria cells for, $40 \mathrm{~mL}$ bacterial culture was harvested by centrifugation at 10,000 rpm for $15 \mathrm{~min}$ at $4{ }^{\circ} \mathrm{C}$, and the cell pellet was washed by $5 \mathrm{~mL}$ deionized water three times at $4{ }^{\circ} \mathrm{C}$. The cell pellets were resuspended in $500 \mu \mathrm{L}$ deionized water. The bacterial solution was adjusted to $10^{8}-10^{9}$ colony-forming units per $\mathrm{mL}$. The bacterial metabolite was extracted by using $100 \% \mathrm{MeOH}$, and was vortexed for $5 \mathrm{~min}$. After centrifugation for $10 \mathrm{~min}$ at $14,000 \mathrm{rpm}, 200 \mu \mathrm{L}$ of supernatant was taken to be vacuum dried at room temperature. The dry sample was reconstituted in $100 \mu \mathrm{L}$ of $80 \% \mathrm{MeOH}$ and then centrifuged at $14,000 \mathrm{rpm}$ for 10 min. The supernatant was collected and subjected to LCESI-MS analysis of positive or negative ion mode. The LCESI-MS system consisted of an ultra-performance liquid chromatography (UPLC) system (Ultimate 3000 RSLC, Dionex) and an electrospray ionization (ESI) source of quadrupole time-of-flight (TOF) mass spectrometer (maXis HUR-QToF system, Bruker Daltonics). The acquired data were processed by TargetAnalysis and DataAnalysis software (Bruker Daltonics) with summarized in an integrated area of signals. Found compounds were selected with the tolerance of LC peaks within 0.3 min and area higher than 1000 counts from established compound identities. The fold change of the metabolite was determined by amount compared to wild-type EHEC EDL933. Student's $t$-test was used to determine the significance of the differences $(P<0.05)$

\section{Real-time quantitative RT-PCR}

Bacteria were cultivated in LB broth at $37^{\circ} \mathrm{C}$ for $16-18 \mathrm{~h}$ with shaking at $220 \mathrm{rpm}$. The overnight bacterial cultures were diluted 1:1000 into fresh $\mathrm{LB}$ broth at $37^{\circ} \mathrm{C}$ with shaking at $220 \mathrm{rpm}$ until cells reached log-phase growth (O.D.600 value is 0.4-0.6). Bacterial cultures were treated with RNAprotect Bacteria Reagent (Qiagen) to stabilize total RNA in bacteria, and total RNA was purified by RNeasy minikit and DNA was removed with on-column DNase digestion (Qiagen). Purified RNAs were quantified with a NanoDrop spectrophotometer, and were reverse transcribed into complementary DNA with M-MLV reverse transcriptase (Promega) using random hexamer primers. Quantitative real time-PCR was performed with 7500 Fast Real-Time PCR system (Applied Biosystems) using SYBR green PCR Master Mix (Roche). RNA polymerase subunit A, rpoA, was used as an internal control, and relative transcriptional expression of each gene was normalized to the level of rpoA. The fold-change of gene expression was determined via $\Delta \Delta \mathrm{Ct}$ values compared to wild-type EHEC EDL933. Student's $t$-test was used for statistical analysis, and $P$ values less than 0.05 were regarded as statistically significant. The primers used in qRT-PCR are listed in Table S4.

\section{Proteomic analysis \\ Sample preparation}

Three bacteria strains, EDL933, EDL933: $\Delta s d h A$, or EDL933: $\Delta s d h A-p W F 134$, were cultured in LB broth at 37 ${ }^{\circ} \mathrm{C}$ for $16-18 \mathrm{~h}$ with agitation at $220 \mathrm{rpm}$. The overnight bacterial cultures were diluted 1:50 into fresh LB broth and cultured to an O.D.600 of 2.0 at $37^{\circ} \mathrm{C}$ with shaking $(220 \mathrm{rpm})$. Bacterial cells were collected from $5 \mathrm{~mL}$ of bacterial culture by centrifugation at $9000 \mathrm{rpm}$ for $10 \mathrm{~min}$ at $4{ }^{\circ} \mathrm{C}$, and were then washed with $10 \mathrm{~mL} 10 \mathrm{mM}$ Tris$\mathrm{HCl}, 5 \mathrm{mM}$ magnesium acetate $(\mathrm{pH} 8.0)$, followed by centrifugation at $9000 \mathrm{rpm}$ for $10 \mathrm{~min}$ at $4{ }^{\circ} \mathrm{C}$. The wash procedures were repeated another four times in order to prevent the proteins from LB broth interfering with the bacterial proteomes. Three independent biological repeats were prepared for each bacterial strain. The final numbers of bacteria were between $10^{8}$ and $10^{9}$ colony-forming units per milliliter. The E. coli samples were extracted by ExtractPRO Protein Extraction Reagent (Visual Protein, Taiwan) and re-suspended directly in RIPA buffer. Finally, the supernatant was kept at $-80^{\circ} \mathrm{C}$.

\section{Gel electrophoresis}

The concentration of protein was determined by the BCA method, and protein samples were analyzed by $12.5 \%$ SDS-PAGE. After electrophoresis, the gels were stained with VisPRO 5-min protein stain kit (Visual Protein, Taiwan). After staining, the gels were washed in Milli-Q water.

\section{In-gel digestion}

The gel lanes corresponding to the bacterial proteins were cut into five slices, and each slice was processed for in-gel digestion according to the method of Shevchenko ${ }^{25}$. Briefly, slices were washed/dehydrated three times in 50 $\mathrm{mM} \mathrm{ABC} \mathrm{(ammonium} \mathrm{bicarbonate} \mathrm{pH}$ 7.9)/50 mM ABC $+50 \%$ ACN (acetonitrile). Subsequently, cysteine bonds were reduced with $10 \mathrm{mM}$ dithiothreitol for $1 \mathrm{~h}$ at $56^{\circ} \mathrm{C}$ and alkylated with $50 \mathrm{mM}$ iodoacetamide for $45 \mathrm{~min}$ at 


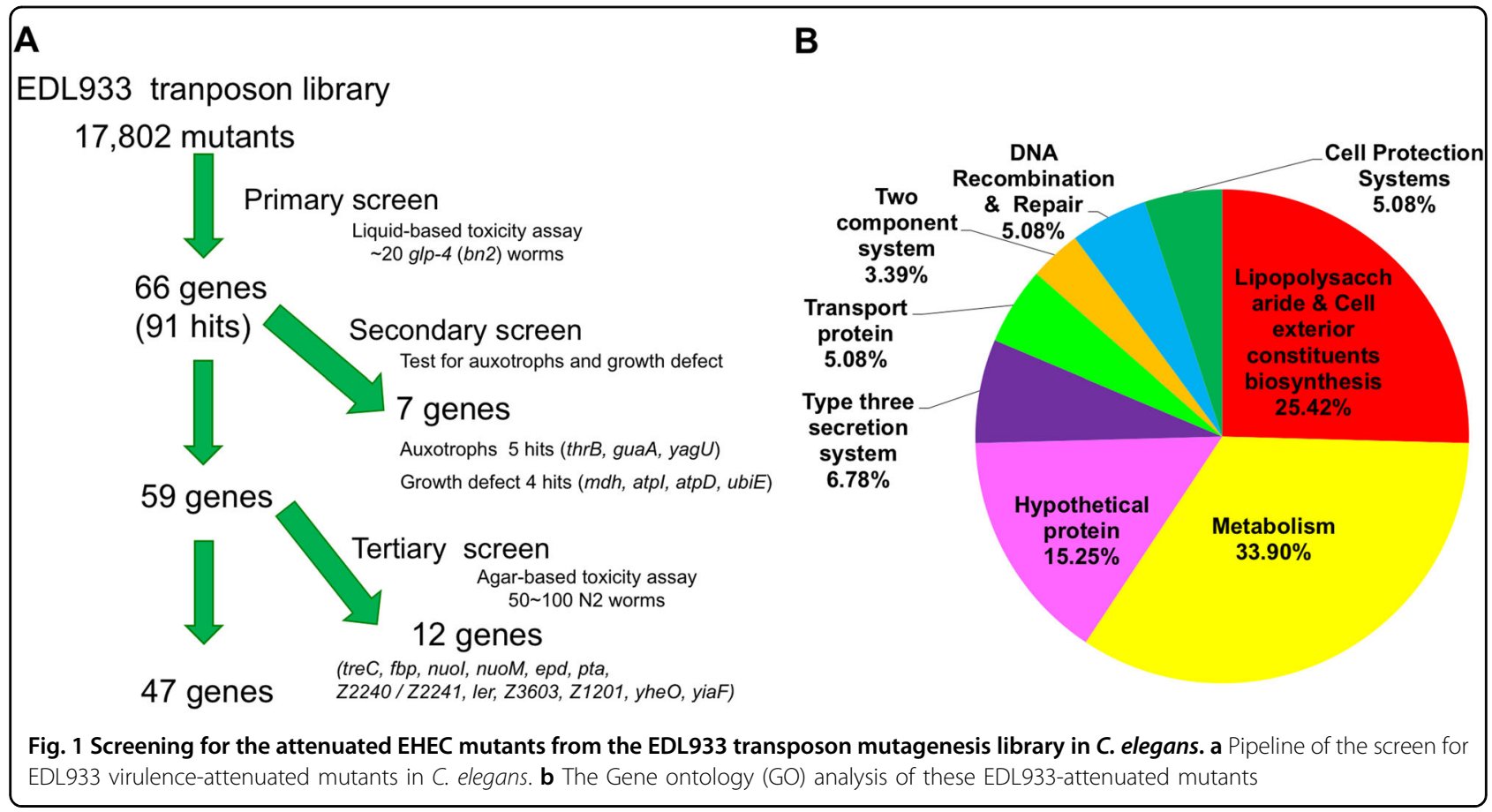

room temperature (RT) in the dark. After two subsequent wash/dehydration cycles, the slices were dried for $10 \mathrm{~min}$ in a vacuum centrifuge (ThermoFisher, Breda, The Netherlands) and incubated overnight with $6.25 \mathrm{ng} / \mu \mathrm{L}$ trypsin in $50 \mathrm{mM} \mathrm{ABC}$ at $25^{\circ} \mathrm{C}$. Peptides were extracted once in $100 \mu \mathrm{L}$ of $1 \%$ formic acid and subsequently twice in $100 \mu \mathrm{L}$ of $50 \% \mathrm{ACN}$ in $5 \%$ formic acid. The volume was reduced to $50 \mu \mathrm{L}$ in a vacuum centrifuge prior to LC-MS/ MS analysis.

\section{Nano-LC separation and mass spectrometry}

Peptides were separated using an Ultimate 3000 nanoLC system (Dionex LC-Packings, Amsterdam, The Netherlands) equipped with a $20 \mathrm{~cm} \times 75 \mu \mathrm{m}$ i.d. fused silica column custom packed with $3 \mu \mathrm{m} 120$ A ReproSil Pur C18 aqua (Dr. Maisch, GMBH, Ammerbuch-Entringen, Germany). After injection, peptides were trapped at $30 \mu \mathrm{L} / \mathrm{min}$ on a $5 \mathrm{~mm} \times 300 \mu \mathrm{m}$ i.d. Pepmap C18 cartridge (Dionex LCPackings, Amsterdam, The Netherlands) at 2\% buffer B (buffer A, 0.05\% formic acid in MQ; buffer B, 80\% $\mathrm{ACN}$ and $0.05 \%$ formic acid in MQ) and separated at 300 $\mathrm{nL} / \mathrm{min}$ in a $10-40 \%$ buffer B gradient in $60 \mathrm{~min}$. Eluting peptides were ionized at $1.7 \mathrm{kV}$ in a Nanomate Triversa Chip-based nanospray source using a Triversa LC coupler (Advion, Ithaca, NJ). Intact peptide mass spectra and fragmentation spectra were acquired on a LTQFT hybrid mass spectrometer (Thermo Fisher, Bremen, Germany). Intact masses were measured at a resolution of 50,000 in the ICR cell using a target value of $1 \times 106$ charges. In parallel, following an FT prescan, the top 5 peptide signals (charge-states $2+$ and higher) were submitted to MS/MS in the linear ion trap ( $3 \mathrm{amu}$ isolation width, $30 \mathrm{~ms}$ activation, $35 \%$ normalized activation energy, $Q$-value of 0.25 and a threshold of 5000 counts). Dynamic exclusion was applied with a repeat count of 1 and an exclusion time of $30 \mathrm{~s}$.

\section{Database search}

MS/MS spectra were searched against $E$. coli using Sequest (version 27, rev 12), which is part of the BioWorks 3.3 data analysis package (Thermo Fisher, San Jose, CA). MS/MS spectra were searched with a maximum allowed deviation of $10 \mathrm{ppm}$ for the precursor mass and $1 \mathrm{amu}$ for fragment masses. Methionine oxidation and cysteine carboxamidomethylation were allowed as variable modifications, two missed cleavages were allowed and the minimum number of tryptic termini was 1 . After the database search, the DTA and OUT files were imported into Scaffold (versions 1.07 and 2.01) (Proteomesoftware, Portland, OR). Scaffold was used to organize the data and to validate peptide identifications using the Peptide Prophet algorithm and only identifications with a probability $>95 \%$ were retained. Subsequently, the Protein-Prophet algorithm was applied and protein identifications with a probability of $>99 \%$ with 2 peptides in at least one of the samples were retained. Proteins that contained similar peptides and could not be differentiated based on MS/MS analysis alone were grouped. For each protein identified, the number of spectral counts (the number of MS/MS associated with an identified protein) is listed in Supplemental Table S5. 

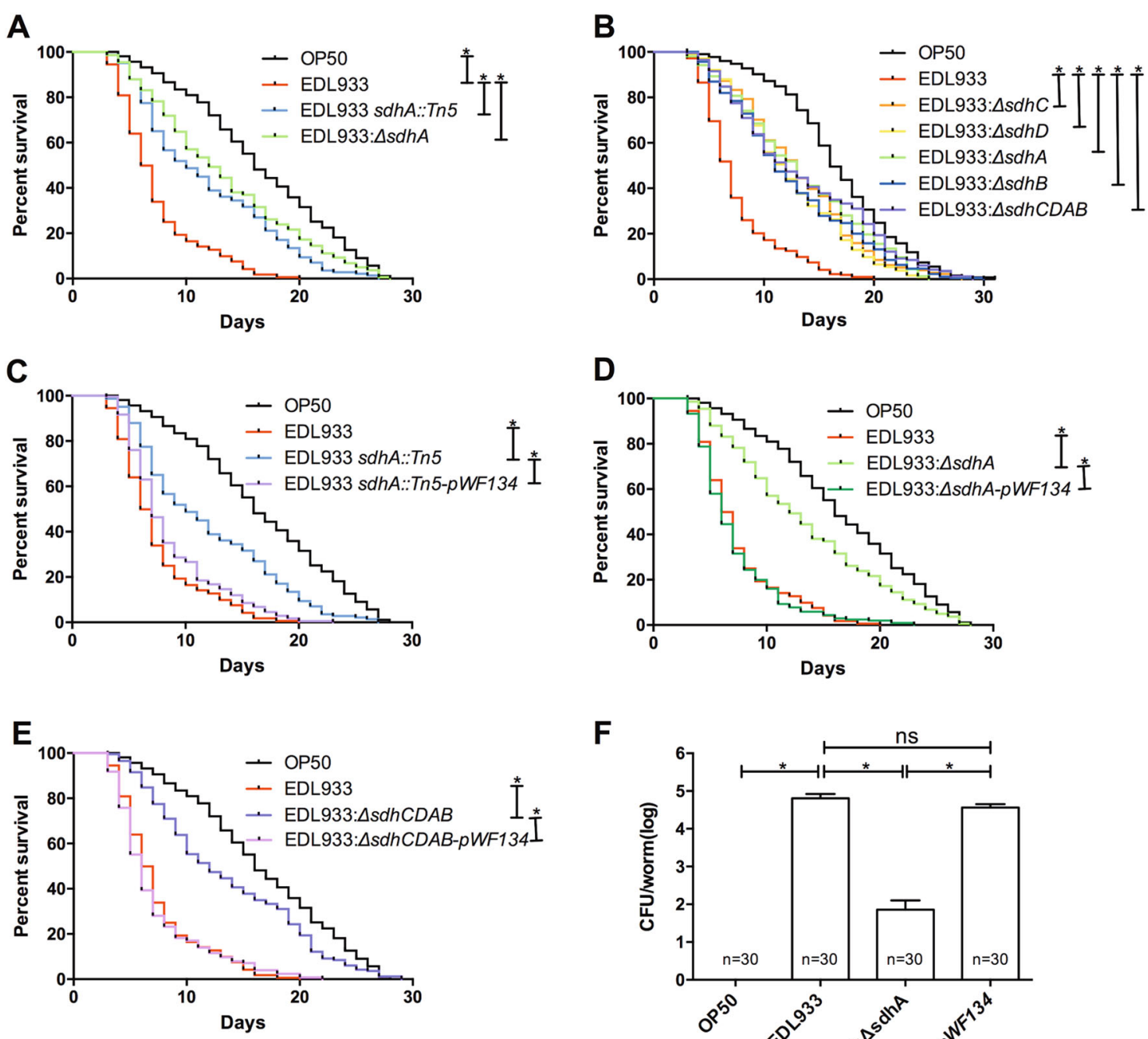

G

$\mathbf{F}$
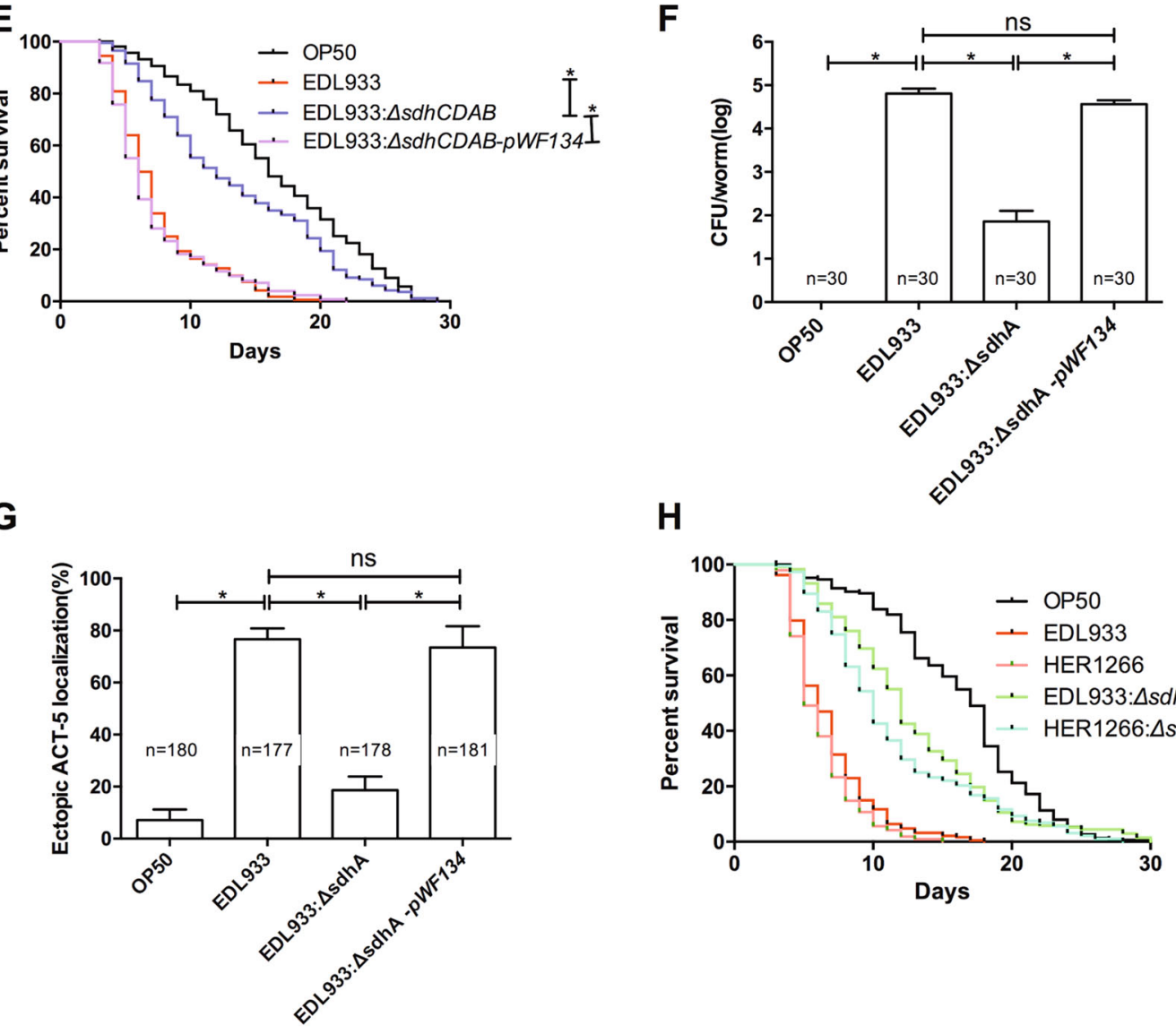

H

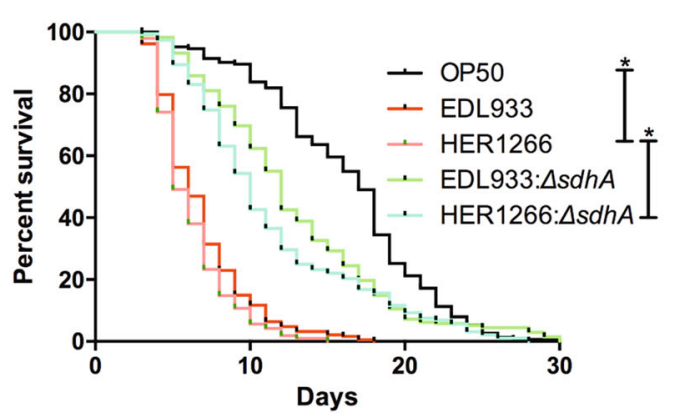

Fig. 2 (See legend on next page.) 


\begin{abstract}
(see figure on previous page)
Fig. 2 Effect of the sdhA mutation on the pathogenicity of EHEC in C. elegans. a The survival of N2 worms fed with E. coli strain OP50, wild-type EHEC strain EDL933, the isogenic transposon-generated mutant strain EDL933 sdhA::Tn5, and the isogenic sdhA deletion mutant strain EDL933: $\triangle$ sdhA were examined. The EDL933 sdhA::Tn5 strain (median N2 lifespan $=10.5 \pm 1.5$ days, $P<0.0001$ ) and the EDL933: $\Delta s d h A$ strain (median N2 lifespan $=$ $12.0 \pm 2.0$ days, $P<0.0001$ ) were both significantly less toxic than the wild-type EDL933 strain (median N2 lifespan $=6.5 \pm 0.5$ days). $\mathbf{b}$ The survival of N2 worms fed with OP50, wild-type EDL933, and EDL933 with deletion of $s d h C$, sdhA, sdhD, sdhB, or sdhCDAB operon were examined. Deletion of sdhC (EDL933: $\triangle s d h C$ (median N2 lifespan $=13.0 \pm 2.0$ days, $P<0.0001$ ), $s d h D$ (EDL933: $\triangle s d h D$ ) (median N2 lifespan $=11.5 \pm 1.5$ days, $P<0.0001$ ), sdhB $(E D L 933: \triangle s d h B)$ (median N2 lifespan $=12.0 \pm 1.0$ days, $P<0.0001)$ genes, and the entire $s d h C D A B$ operon (EDL933: $\triangle s d h C D A B)$ (median N2 lifespan $=$ $12.5 \pm 2.5$ days, $P<0.0001$ ) were all significantly less toxic than the wild-type EHEC strain EDL933 (EDL933) (median N2 lifespan $=6.75 \pm 0.25$ days). c-e The survival of N2 worms fed with sdhA transposon-generated mutant EDL933 sdhA.:Tn5, sdhA deletion mutant (EDL933: $\triangle$ sdhA), sdhCDAB deletion mutant (EDL933: $\triangle S d h C D A B)$, and isogeneic strains rescued by the transformation of the $s d h C D A B$ operon expression plasmid $p W F 134$

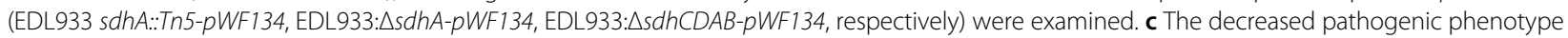
in the EDL933 sdhA.:Tn5 (median N2 lifespan = $10.5 \pm 1.5$ days) was restored by the sdhA gene complementation EDL933 sdhA.:Tn5-pWF134 (median N2 lifespan $=7.0 \pm 0.1$ days, $P<0.0001$ ). $\mathbf{d}$ EDL933: $\triangle$ sdhA (median N2 lifespan $=12.00 \pm 2.00$ days) and EDL933: $\triangle$ sdhA-pWF134 (median N2 lifespan $=$ $6.0 \pm 1.0$ days, $P<0.0001$ ). e EDL933: $\triangle s d h C D A B$ (median N2 lifespan $=12.5 \pm 2.5$ days) and EDL933: $\triangle$ sdhCDAB-pWF134 (median N2 lifespan $=5.5 \pm$ 0.5 days, $P<0.0001$ ). The median lifespan of N2 worms fed with wild-type EDL933 was $6.5 \pm 0.5$ days. $\mathbf{f}$ The number of bacteria colonized in $C$. elegans was determined by the colony-forming units (CFUs) assay from three independent experiments. The average CFU per infected worm fed with the sdhA deletion mutant was significantly decreased $\left(7.10 \times 10^{1}, P<0.0001\right)$ compared to that of the wild-type EDL933 infected animals $\left(6.43 \times 10^{4}\right)$. The average CFU per infected worm fed with the $s d h A$ gene complementation strain $\left(3.65 \times 10^{4}, P=0.1\right)$ was similar to that of the wild-type EDL933 infected animals. The asterisk denotes statistically significant $(P<0.0001)$ examined by the $t$-test, and error bars indicate the SEM (standard error of mean) of three independent experiments. The total numbers of infected animals examined in each group are indicated by $n$. $\mathbf{g}$ Quantification of the ectopic mCherry::ACT-5 signal in GK454 nematodes exposed to OP50, EDL933, sdhA deletion mutant (EDL933: $\triangle$ sdhA), or sdhA gene complementation strain (EDL933: $\triangle$ sdhA-pWF134) at $20^{\circ} \mathrm{C}$ for 4 days. The asterisk denotes statistically significant $(P<0.0001)$ examined by the $t$-test, and error bars indicate the SEM of three independent experiments. The total numbers of infected animals examined in each group are indicated by $n$. $\mathbf{h}$ Survival of N2 worms fed with the E. coli O157:H7 strain HER1266 and the isogenic strain with sdhA deletion. Deletion of sdhA in HER1266 (HER1266: $\triangle s d h A)$ confers the attenuated toxic phenotype (median lifespan of N2 animals $=10.0 \pm 0.1$ days, $P<0.0001$ ). The median lifespan of N2 worms fed with wild-type HER1266 was $5.50 \pm 0.5$ days
\end{abstract}

\section{Data analysis}

All experiments were performed a minimum of three times independently. Two values were compared with a paired $t$-test, and three or more values of one independent variable was conducted with matched one-way ANOVA with Tukey's method and more than two independent variables by two-way ANOVA with the Bonferroni post test. All data analysis was performed using SPSS, v. 13.0 (SPSS, Chicago, IL). Statistical significance was set at $P<$ 0.05 .

\section{Results \\ Functional genomic screening of the EHEC transposon library in C. elegans}

To identify genes involved in the EHEC pathogenicity in vivo, we conducted a global mutational study by using the EHEC strain EDL933 Tn5 transposon library. After the primary screen of the 17,802 EDL933 mutants in liquid-based killing assay, 91 hits with a decreased pathogenic phenotype toward C. elegans were identified. The transposition sites of $\operatorname{Tn} 5$ in these 91 clones were determined (Fig. 1a and Supplemental Table S6). These 91 hits included mutations in 66 genes. We eliminated 4 hits with growth defect and 5 hits with auxotrophic phenotype after the secondary screen. It has been reported that Pseudomonas aerginosa killing C. elegans in liquid-based killing assay uses different virulence factors from plate-based assay ${ }^{26}$. We therefore conducted plate- based killing assays to examine the survival rate of $C$. elegans fed with these identified EDL933 mutants. Among the 59 genes, mutations of 47 genes conferred attenuated phenotype after this tertiary screen, which reconfirmed that these genes are required for full EHEC toxicity in both liquid-based and plate-based killing. Interestingly, the functions of these 59 genes were diverse (Fig. 1b). Moreover, several bacterial factors found in our genetic screening have also been reported as important virulence factors for EHEC, including ler and eae, and $h f q^{27,28}$. Given that the genes involved in metabolism represented the major part of our hits, we turned our attention to the genes in this category.

sdhA is required for the pathogenicity of EHEC in C. elegans

We noted that several genes in central metabolism, especially the succinate dehydrogenase $(s d h)$ genes, are required for the pathogenesis of EHEC in C. elegans. The transposon mutant $s d h A:: T n 5$, strain YQ413 (ED97-A-1 in Supplemental Table S6) conferred a significant decrease in the virulence toward C. elegans (Fig. 2a). In order to reconfirm that mutation in $s d h A$ attenuates the toxicity of EHEC, an isogenic deletion mutant (EDL933: $\Delta s d h A$ ) was generated. The $s d h A$ deletion significantly attenuated the pathogenicity of EHEC compared to the parental wild-type EDL933 (Fig. 2a). Of note, the $s d h A$ mutants, including the transposon and deletion mutants, all showed no growth defect (Supplemental Figure S2), 

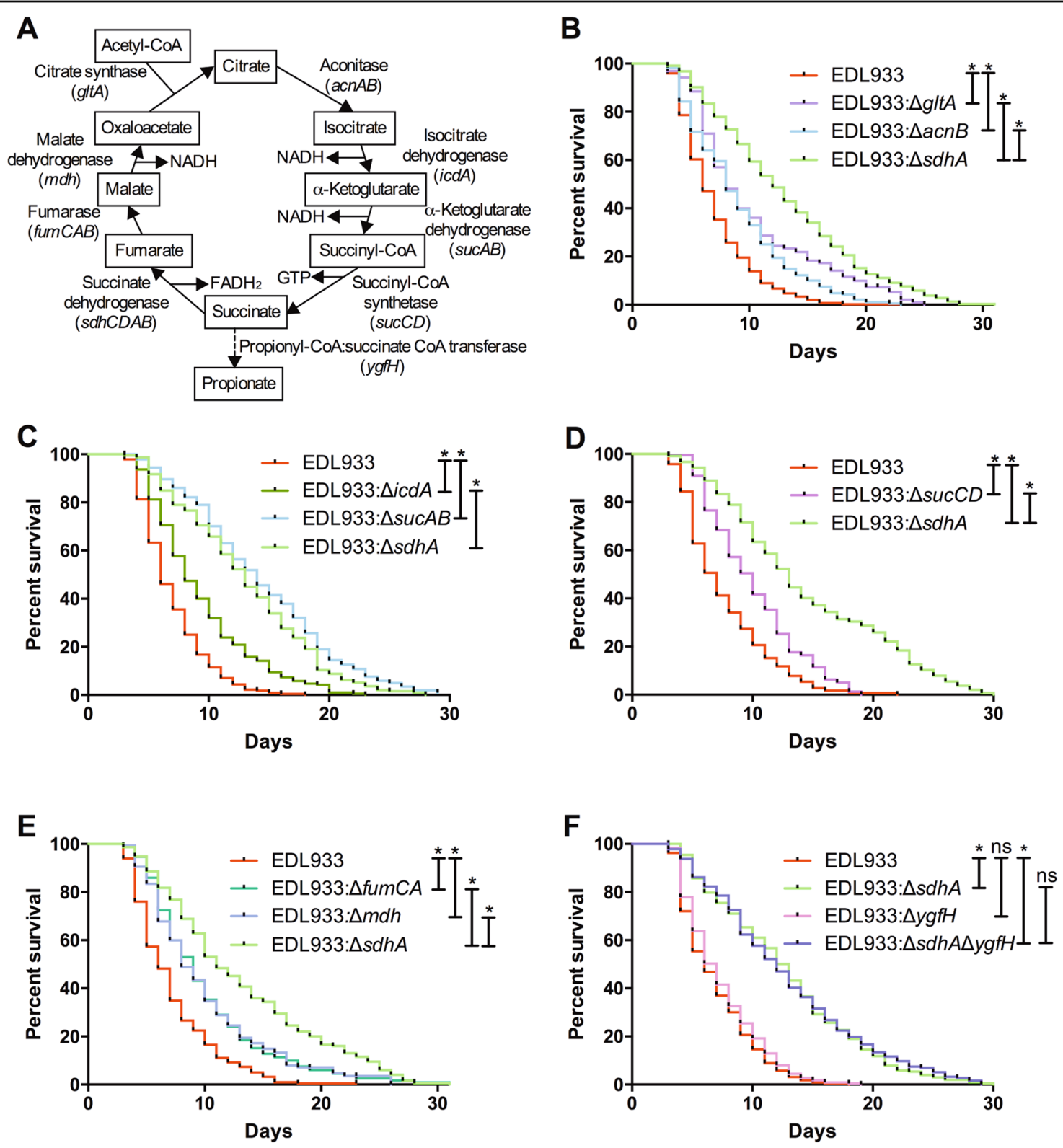

Fig. 3 (See legend on next page.)

which suggested that $s d h A$ may play unidentified roles in EHEC pathogenicity. Hence, we turned our focus to the mechanism of $s d h A$ in the regulation of EHEC infection.

The Sdh complex consists of four subunits and is encoded by the $s d h C D A B$ operon $^{29}$. We found that disruptions in any genes of the complex, including $s d h C$, $s d h D, s d h A$, and $s d h B$, and the entire $s d h C D A B$ operon all conferred attenuated toxicity of EHEC toward $C$. elegans (Fig. 2b). These data suggest that Sdh activity is required for the pathogenesis of EHEC. In order to reconfirm the role of $\mathrm{Sdh}$ in the virulence of EHEC, the $s d h C D A B$ operon expression plasmid $p W F 134$ was generated for complementation of these mutants. Complementation of the $s d h$ mutations by the $p$ WF134 reversed the attenuated toxicity of the transposongenerated $s d h A$ mutant EDL933 sdhA::Tn5, (Fig. 2c), the $s d h A$ deletion mutant EDL933: $\Delta s d h A$ (Fig. 2d), and the $s d h C D A B$ operon deletion mutant EDL933: $\triangle s d h C D A B$ (Fig. 2e). Taken together, our genetic analysis demonstrated that the $s d h$ genes, including $s d h A$, are required for the virulence of EHEC strain EDL933 in C. elegans. 


\begin{abstract}
(see figure on previous page)
Fig. 3 Effect of the incomplete TCA cycle and succinate metabolism on the virulence of EHEC. a A diagram of the enzymes (genes) and metabolites of the TCA cycle and the alternative metabolic path of succinate through YgfH. $\mathbf{b}$ The survival of N2 worms fed with the wild-type EHEC

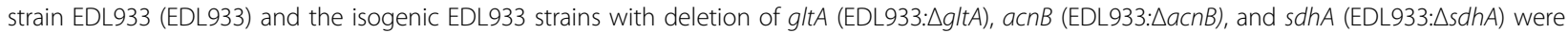
examined. Deletions of gltA (median N2 lifespan $=8.5 \pm 0.7$ days, $P<0.0001$ ), $a c n B$ (median N2 lifespan $=8.0 \pm 0.1$ days, $P<0.0001$ ), and sdhA (median N2 lifespan $=12.0 \pm 2.2$ days, $P<0.0001$ ) all conferred the attenuated toxic phenotype compared to the wild-type EDL933 (the median N2 lifespan $=6.0 \pm 0.1$ days). Moreover, EDL933: $\triangle s d h A$ mutant was significantly less toxic compared to the EDL933: $\triangle g \mid t A(P<0.0001)$ and EDL933: $\triangle a c n B$

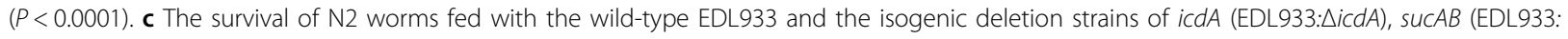
$\triangle s u C A B$ ), and $s d h A$ (EDL933: $\triangle s d h A$ ) were examined. Deletions of icdA (median N2 lifespan $=9.0 \pm 1.4$ days, $P<0.005$ ), sucAB (median N2 lifespan $=$ $13.5 \pm 2.1$ days, $P<0.0001$ ), and $s d h A$ (median N2 lifespan $=13.5 \pm 2.1$ days, $P<0.0001$ ) all conferred the attenuated toxic phenotype compared to the wild-type EDL933 (the median N2 lifespan $=6 \pm 0.2$ days). The EDL933: $\triangle$ sdhA mutant was significantly less toxic compared to both the EDL933: $\triangle i c d A(P<0.0001)$ and the EDL933: $\triangle$ suCAB mutant $(P<0.05)$. $\mathbf{d}$ The survival of $\mathrm{N} 2$ worms fed with the wild-type EDL933 and the isogenic deletion strains of sucCD (EDL933: $\triangle s u C C D)$ and sdhA (EDL933: $\triangle s d h A)$ were examined. Deletion of sucCD (median N2 lifespan $=9.0 \pm 1.0$ days, $P<0.0001)$ and sdhA (median N2 lifespan $=11.3 \pm 1.5$ days, $P<0.0001$ ) conferred the attenuated toxic phenotype compared to the wild-type EDL933 (the median N2 lifespan $=6.3 \pm 0.5$ days). Moreover, EDL933: $\triangle$ sdhA mutant was significantly less toxic compared to the EDL933: $\triangle s u c C D(P<0.0001)$. e The survival of

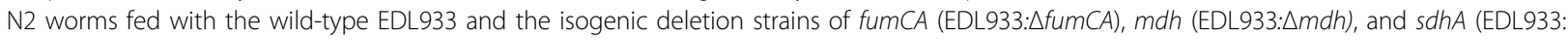
$\triangle s d h A$ ) were examined. Deletions of fumCA (median N2 lifespan $=9.0 \pm 0.1$ days, $P<0.0001$ ), mdh (median N2 lifespan $=8.5 \pm 0.5$ days, $P<0.0001$ ), and sdhA (median N2 lifespan $=10.5 \pm 0.5$ days, $P<0.0001$ ) all conferred the attenuated toxic phenotype compared to the wild-type EDL933 (median

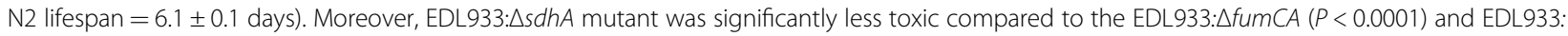
$\triangle m d h(P<0.0001)$. $f$ The survival of N2 worms fed with the wild-type EDL933 and the isogenic deletion strains of ygfH (EDL933: $\triangle y g f H)$, sdhA (EDL933: $\triangle s d h A$ ), and the $y g f H$ and $s d h A$ double mutant (EDL933: $\triangle y g f H \triangle s d h A$ ) were examined. Deletion of $y g f H$ (median N2 lifespan $=6.3 \pm 0.4$ days, $P=0.102$ ) did not attenuate its toxicity. Although deletion of the ygfH and sdhA double mutant (EDL933: $\Delta y g f H \Delta s d h A$, median N2 lifespan $=11.5 \pm 0.7$ days, $P<$ 0.0001) conferred the attenuated toxic phenotype compared to the wild-type EDL933 (the median N2 lifespan $=5.0 \pm 0.5$ days), the virulence of

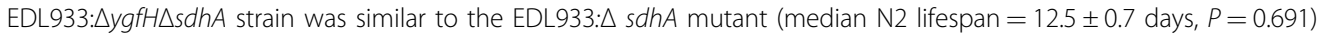

Our previous study demonstrated that EHEC can colonize and induce abnormal microvillus morphology, a biomarker of the $\mathrm{A} / \mathrm{E}$ lesion, in the intestine of $C$. ele$g_{a n s}{ }^{19}$. Here we demonstrated that mutation of the EHEC sdhA gene significantly reduced its colonization in C. elegans (Fig. 2f). Moreover, complementation by $p$ WF134 restored this phenotype of the EDL933: $\Delta s d h A$ mutant. The abnormal microvillus morphology induced by EHEC can be detected by the mislocalization of the intestine microvillar Actin-5 that is normally expressed on the apical site and in the microvilli of intestinal cells ${ }^{19}$. Compared to the wild-type EDL933, the EHECinduced ectopic microvillar actin localization was significantly diminished in the EDL933: $\Delta s d h A$ infected $C$. elegans animals (Fig. 2g). Moreover, complementation of the $s d h A$ mutation by $p W F 134$ significantly rescued the phenotype. Together, our results demonstrated that $s d h A$ mutation decreased the virulence of EHEC toward C. elegans.

In order to test whether $s d h A$ is also a virulence determinant for the other EHEC strain, an isogenic mutant with $s d h A$ deletion in the EHEC strain HER1266 (HER1266: $\Delta s d h A$ ) was generated. Disruption of the $s d h A$ gene also attenuated the toxicity of HER1266 in C. elegans (Fig. 2h). However, C. elegans fed on OP50: $\Delta s d h A$ exhibited a similar survival curve to that of the worms fed on wild-type OP50, the normal food source of C. elegans (Supplemental Figure S4B). Together, our data indicated that $s d h A$ is a specific virulence determinant and required for EHEC infection in C. elegans.

\section{Deletions of the TCA cycle genes confer the attenuated virulence of EHEC}

Sdh converts succinate to fumarate in the TCA cycle (Fig. 3a). In addition to the $s d h C D A B$ operon, the glt $A$, $\operatorname{acn} A B$, icd $A, \operatorname{suc} A B$, sucCD, fum $C A B$, and $m d h$ genes encode for the other enzymes in the cycle. To examine whether the TCA cycle is required for the virulence regulation of EHEC, we interrupted the TCA cycle by generating isogeneic deletion mutants of these genes. Our data demonstrated that deletion of these genes significantly attenuated the toxicity of EHEC toward C. elegans (Fig. 3b-e). Interestingly, the effects of disruption of these TCA cycle genes ( $g l t A, \operatorname{acn} B$, icdA, sucAB, sucCD, fum $C A$, and $m d h$ respectively) in EHEC virulence attenuation were all significantly less (all $P<0.05)$ than the disruption of the $s d h A$ gene. These results suggested that disruption of the TCA cycle accounts only partly for the attenuated virulent phenotype of the $s d h A$ mutant. Nevertheless, our data suggested that the TCA cycle plays an important role in the pathogenesis of EHEC.

Except for Sdh, which oxidized succinate to form fumarate, propionyl-CoA:succinate $\mathrm{CoA}$ transferase, encoded by the $y g f H$ gene, can also decarboxylate succinate to form propionate (Fig. 3a). However, we found that disruption of the $y g f H$ gene had no effect on the virulence of EHEC toward C. elegans (Fig. 3f). Moreover, the $y g f H$ and $s d h A$ double mutant conferred the same attenuated toxic phenotype as the $s d h A$ single mutant. Our genetic data indicated that $y g f H$ is not required for the EHEC virulence in $C$. elegans. Nevertheless, our results 
reconfirmed that $s d h A$ is required for full toxicity of EHEC in C. elegans.

\section{EHEC attenuates its toxicity upon anaerobic respiration}

E. coli cells have three metabolic modes of energy metabolism: aerobic respiration, anaerobic respiration, and fermentation. Sdh also serves as the complex II in the aerobic respiratory chain. Given that interruption of the TCA cycle by deletion of $s d h A$ decreases the toxicity of EHEC, we hypothesized that EHEC is more toxic upon oxybiosis. To this end, we constructed isogenic deletion mutants of the genes encoded for the enzymes and regulators in either aerobic respiration or anaerobic metabolism of EHEC, and tested their toxicity toward C. elegans.

Besides Sdh, the NADH dehydrogenase (complex I), encoded by the nuo operon, can transfer electrons to ubiquinone for aerobic respiration. Our results showed that the survival curve of $C$. elegans fed with EDL933: $\Delta$ nиoH was significantly longer than that of animals fed with the wildtype EDL933 (Fig. 4a). Moreover, the ArcAB twocomponent regulatory system is the major regulator of aerobic respiration ${ }^{6,30}$. Our data indicated that the $\operatorname{arc} A B$ double mutant (EDL933: $\triangle \operatorname{arc} A B$ ) strain was significantly less toxic compared to the wild type (Fig. 4a). However, both the EDL933: $\triangle n u o H$ and EDL933: $\triangle \operatorname{arc} A B$ mutants were significantly more toxic than the EDL933: $\Delta s d h A$ mutant. The results suggested that disruption of aerobic respiration partly accounts for the attenuated virulent phenotype of the $s d h A$ mutant. Moreover, we also demonstrated that anaerobic metabolism is dispensable for the full virulence of EHEC in C. elegans (Supplemental Figure S3). Taken together, these results supported the notion that EHEC is more toxic when utilizing aerobic respiration.

To test the hypothesis further, we cultured the EDL933 bacterial cells either aerobically or anaerobically and tested their toxicity toward C. elegans. We observed that the virulence of the wild-type EDL933 and the EDL933: $\Delta s d h A-p W F 134$ strains when cultured anaerobically were both significantly attenuated compared to the same strains cultured aerobically (Fig. 4b). These data support the notion that EHEC is more toxic in aerobic metabolism. Moreover, C. elegans fed on EDL933: $\Delta s d h A$ cells cultured anaerobically exhibited similar survival curves to worms fed on the bacterial cells cultured aerobically (Fig. 4c). Together, our results demonstrated that aerobic respiration plays a role in the regulation of pathogenicity of EHEC in C. elegans, and accounts partly for the decreased virulence of the $s d h A$ mutant.

\section{The depletion of fumarate resulted from sdhA mutation affecting the EHEC toxicity}

A major metabolic function of $\mathrm{Sdh}$ is to convert succinate into fumarate. We surmised that the alteration in the concentrations of related metabolites could

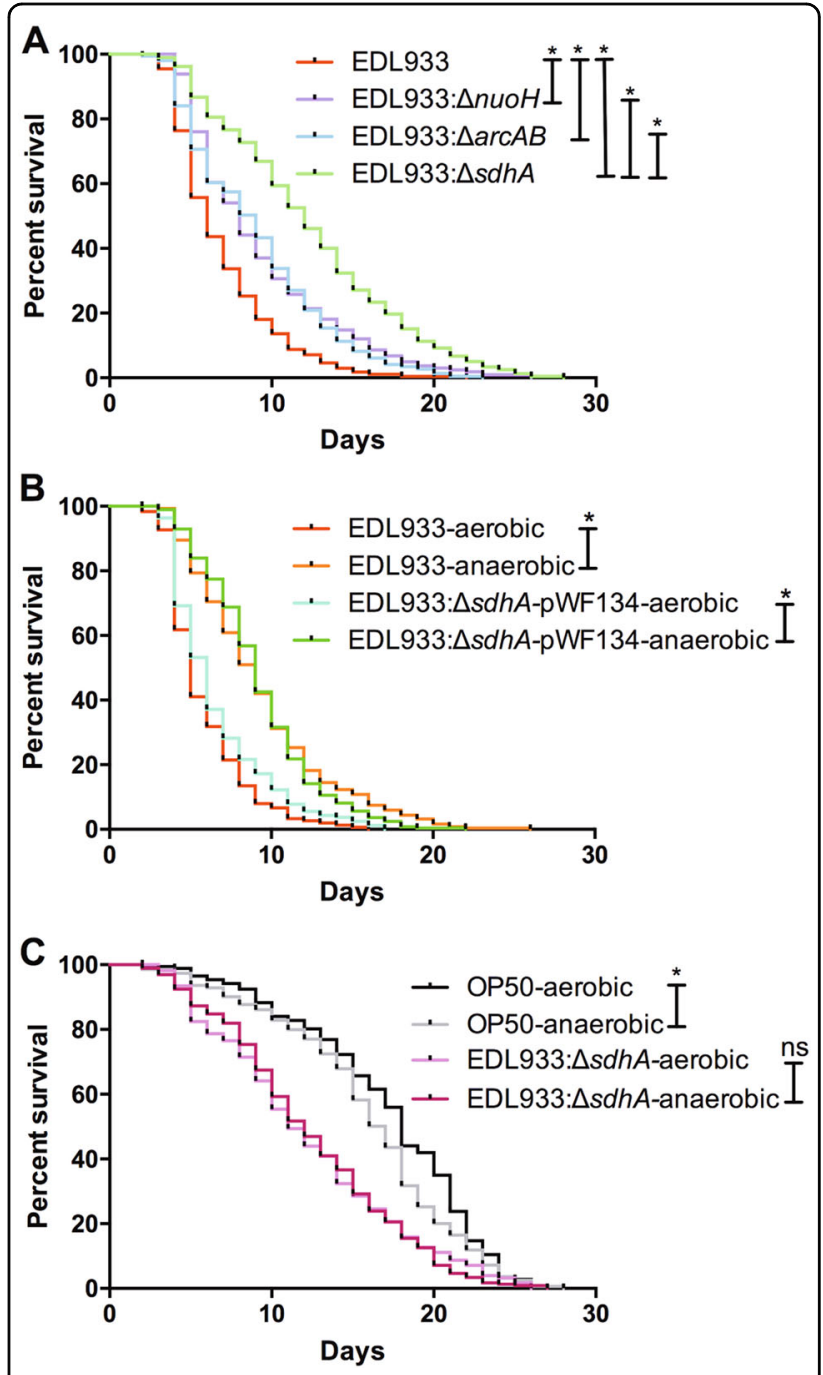

Fig. 4 (See legend on next page.)

potentially influence the toxicity of EHEC. Thus, we first assessed the level of succinate and fumarate in the EDL933: $\Delta s d h A$ mutants. We noted that the levels of succinate in the transposon-generated $s d h A$ mutant [YQ413 (EDL933 sdhA::Tn5)] and the isogenic sdhA deletion mutant (EDL933: $\Delta s d h A$ ) of EDL933 strain were both significantly increased compared to the parental EDL933 strain (Fig. 5a). In contrast, the levels of fumarate in the EDL933 sdhA::Tn5, and the EDL933: $\Delta s d h A$ strains were both significantly decreased compared to the wildtype (Fig. 5b). These data reconfirmed that disruption of the enzyme activity of Sdh in the $s d h A$ mutants alters the concentrations of the related metabolites in EHEC.

We then tested whether accumulation of succinate or depletion of fumarate caused by loss-of-function of $s d h A$ could affect the EHEC pathogenicity. To this end, we 


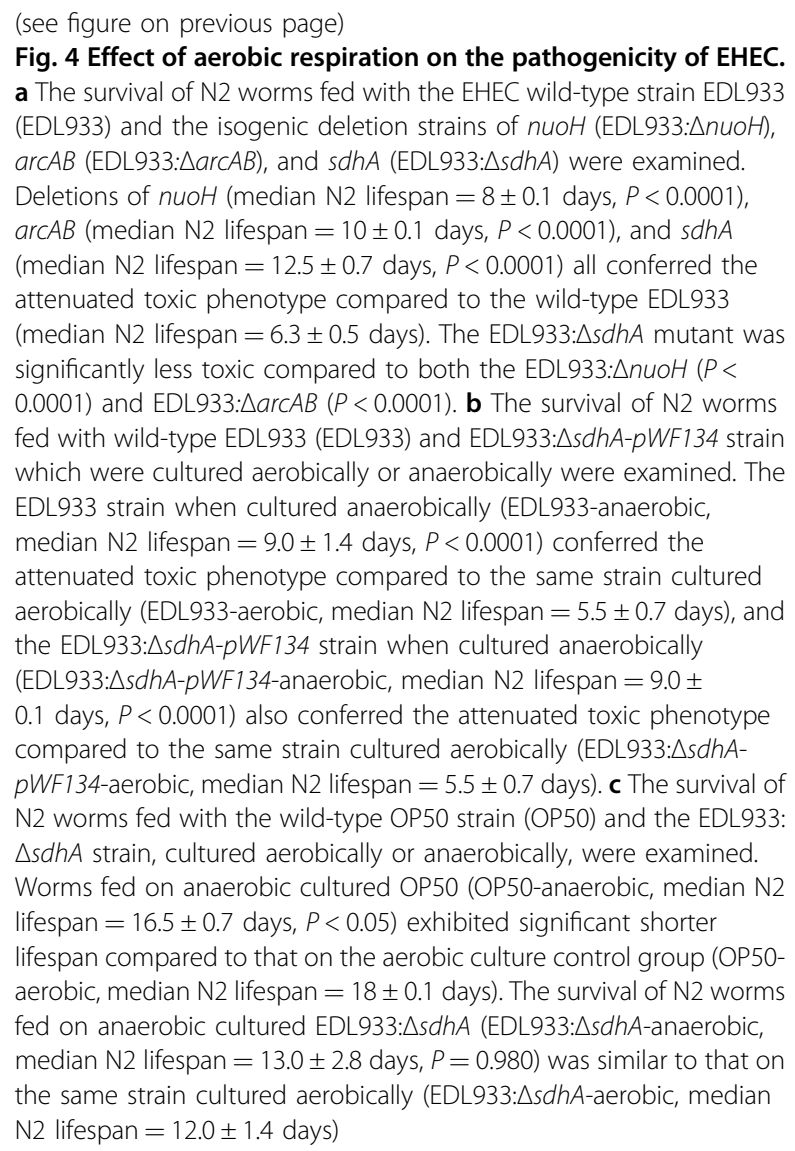

cultured wild-type OP50, OP50: $\Delta s d h A$, wild-type EDL933 and the EDL933: $\Delta s d h A$ mutant with $2.5 \mathrm{mM}$ succinate or $2.5 \mathrm{mM}$ fumarate, and tested their toxicity against $C$. elegans. We noted that while treatment of succinate slightly decreased the toxicity of EHEC against C. elegans, treatment of fumarate in the wild-type EDL933 had no effect on its virulence (Fig. 5c). However, the toxic attenuation of the EDL933: $\Delta s d h A$ mutant was significantly reversed by culture of bacterial cells with fumarate, but not with succinate, compared to the untreated EDL933: $\Delta s d h A$ control (Fig. 5d). The data demonstrated fumarate depletion accounts significantly for the attenuated virulent phenotype of the $s d h A$ mutant. We also demonstrated that the effect of fumarate was on EDL933: $\Delta s d h A$ mutant per se, rather than toward C. elegans (Supplemental Figure S4). Taken together, our data suggest that fumarate may function as a virulent determinant to regulate the pathogenicity of EHEC.

\section{TnaA tryptophanase is a downstream virulence effector of $\operatorname{sdh} A$}

In order to identify the downstream virulence effectors regulated by the activity of $\mathrm{Sdh}$, we deployed a proteomic analysis to characterize the proteomic changes of
EDL933: $\Delta s d h A$ mutant (Supplemental Table S5). Four differentially expressed proteins, which were downregulated in the $s d h A$ mutant as well upregulated in the $s d h A$ complement strain, were identified (Fig. 6a and Table 1). These four proteins are the succinate dehydrogenase flavoprotein subunit SdhA, the succinate dehydrogenase iron-sulfur subunit SdhB, the tryptophanase TnaA, and the molecular protein chaperone DnaJ. The identification of the SdhA and SdhB proteins from our analyses validated the reliability of our proteomic data. In addition, we identified two potential Sdhdependent and downstream virulence modulators, TnaA and DnaJ. Consistent with their expression at the protein level, the transcription levels of tnaA and $d n a J$ genes were significantly reduced in the EDL933: $\Delta s d h A$ mutant compared to the wild-type EDL933 (Fig. 6b). Moreover, the complementation of $s d h A$ mutation by $p W F 134$ transformation as well as fumarate supplement can both restore their mRNA expression in the $s d h A$ mutant, which suggested that the expression of tnaA and $d n a J$ is regulated in a $s d h A$ - and fumarate-dependent manner at the transcriptional level.

Given that $d n a J$ is an essential gene required for $E$. coli growth $^{31}$, we turned our attention to the function tnaA gene in the virulence of EHEC. Our results showed that disruption of the tnaA significantly attenuated the toxicity of EDL933 in C. elegans (Fig. 6c), suggesting that TnaA is also a virulence determinant of EHEC. We noted that the virulence of the EDL933: $\Delta s d h A \Delta t n a A$ strain to C. elegans animals was more significantly decreased than that of the EDL933: $\Delta$ tnaA strain, but is similar to that of the EDL933: $\Delta s d h A$ mutant (Fig. 6d). These genetic epistasis results placed the tnaA downstream and in the same pathway of $s d h A$. Together with our proteomic and qRTPCR analyses, these results demonstrated that the expressions of the TnaA protein and mRNA are $s d h A$-and fumarate-dependent.

\section{Cra is required for fumarate-dependent virulence regulation in EHEC}

Fumarate and succinate belong to the C4-dicarboxylates that are metabolized by bacteria under aerobic or anaerobic conditions ${ }^{32}$. Sensing and uptaking of C4dicarboxylates are achieved by several two-component systems in bacteria. We have tested three putative twocomponent systems for $\mathrm{C} 4$ dicarboxylates regulation, including the DcuSR, DctSR, and DctBD, and found there are not involved in sensing fumarate to regulate the virulence of EHEC (Supplemental Figure S5).

Recently, a transcription factor Cra (FruR) which regulates carbohydrate metabolic enzymes to control sugar concentration in E. coli has been reported to modulate EHEC virulence effectors that are required for formation of $\mathrm{A} / \mathrm{E}$ lesions ${ }^{8,33}$. Given that $\mathrm{Cra}$ has also been reported 

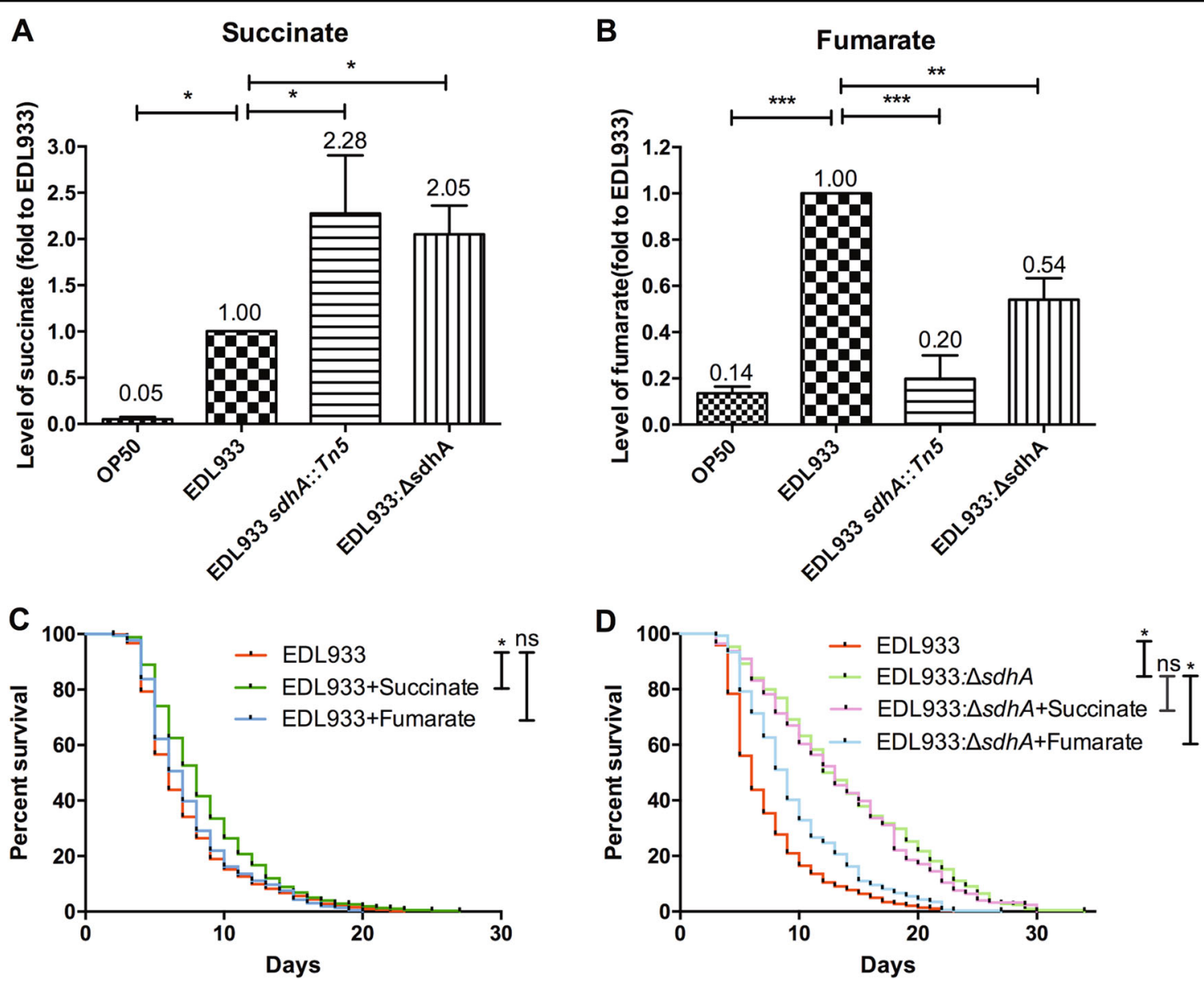

Fig. 5 Effect of metabolite alteration on the pathogenesis of EDL933: $\Delta s d h A$ mutant. a The relative level of succinate in OP50 strain, wild-type

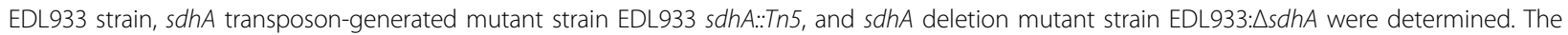
asterisk denotes statistical significance $(P<0.0001)$ as examined by the $t$-test, and error bars indicate the SEM (Standard Error of the Mean) of three independent experiments. b The relative level of fumarate in OP50, EDL933, EDL933 sdhA::Tn5, and EDL933: $\triangle$ sdhA were determined. $\mathbf{c}$ The survival curves of N2 worms fed with the wild-type EDL933 strain only (EDL933), EDL933 cultured in media supplemented with 2.5 mM succinate (EDL933 + Succinate), or $2.5 \mathrm{mM}$ fumarate (EDL933 + Fumarate) were examined. Treatment of succinate in EDL933 strain conferred the attenuated toxic phenotype (EDL933 + Succinate, median N2 lifespan $=7.8 \pm 0.6$ days, $P<0.0001$ ) compared to the untreated wild-type EDL933 strain control (median N2 lifespan $=5.9 \pm 0.3$ days). Treatment of fumarate in EDL933 strain expressed similar toxicity (median N2 lifespan $=6.7 \pm 0.3$ days, $P=0.18$ ) as the untreated wild-type EDL933 strain control (EDL933 + Fumarate, N2 median lifespan $=5.9 \pm 0.3$ days). $\mathbf{d}$ The survival curves of worms fed with

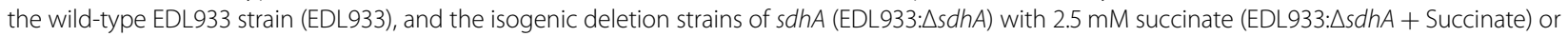

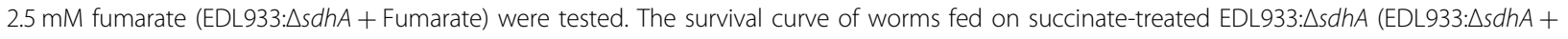
Succinate, median N2 lifespan $=12.5 \pm 2.1$ days, $P=0.927)$ was similar to that on the control EDL933: $\triangle s d h A(E D L 933: \triangle s d h A$, median N2 lifespan $=$ $13.3 \pm 1.5$ days). However, treatment of fumarate in EDL933: $\triangle$ sdhA mutant (EDL933: $\triangle s d h A+$ Fumarate, median N2 lifespan $=8.8 \pm 0.8$ days, $P<$

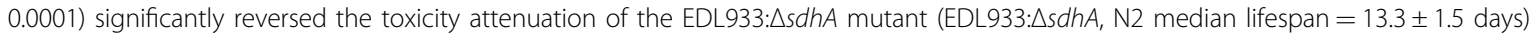

to function as a metabolic flux sensor in $E$. $\operatorname{coli}^{34}$, we tested whether Cra can sense fumarate and restore $s d h A$ mutant toxicity. As shown in Fig. 7a, treatment of $2.5 \mathrm{mM}$ fumarate can fully restore the virulence of the $s d h A$ single mutant. However, this effect of fumarate was totally blocked in the sdhAcra double mutant, suggesting that fumarate rescue of the toxicity of $s d h A$ mutant is Cradependent. Taken together, these findings show that fumarate regulates EHEC toxicity through the Cra transcription factor.

\section{Discussion}

Succinate dehydrogenase (Sdh) complex is involved in both the TCA cycle and the aerobic respiratory chain simultaneously. Here, we demonstrated that disruption of the $s d h A$ gene attenuated the toxicity of EHEC in vivo. Our data also showed that disruptions of the TCA cycle and aerobic metabolism both have effects on the toxicity attenuation of the $s d h A$ mutant. Moreover, we demonstrated that down regulation of the virulence determinant tnaA in part accounts for the attenuated toxicity of the 


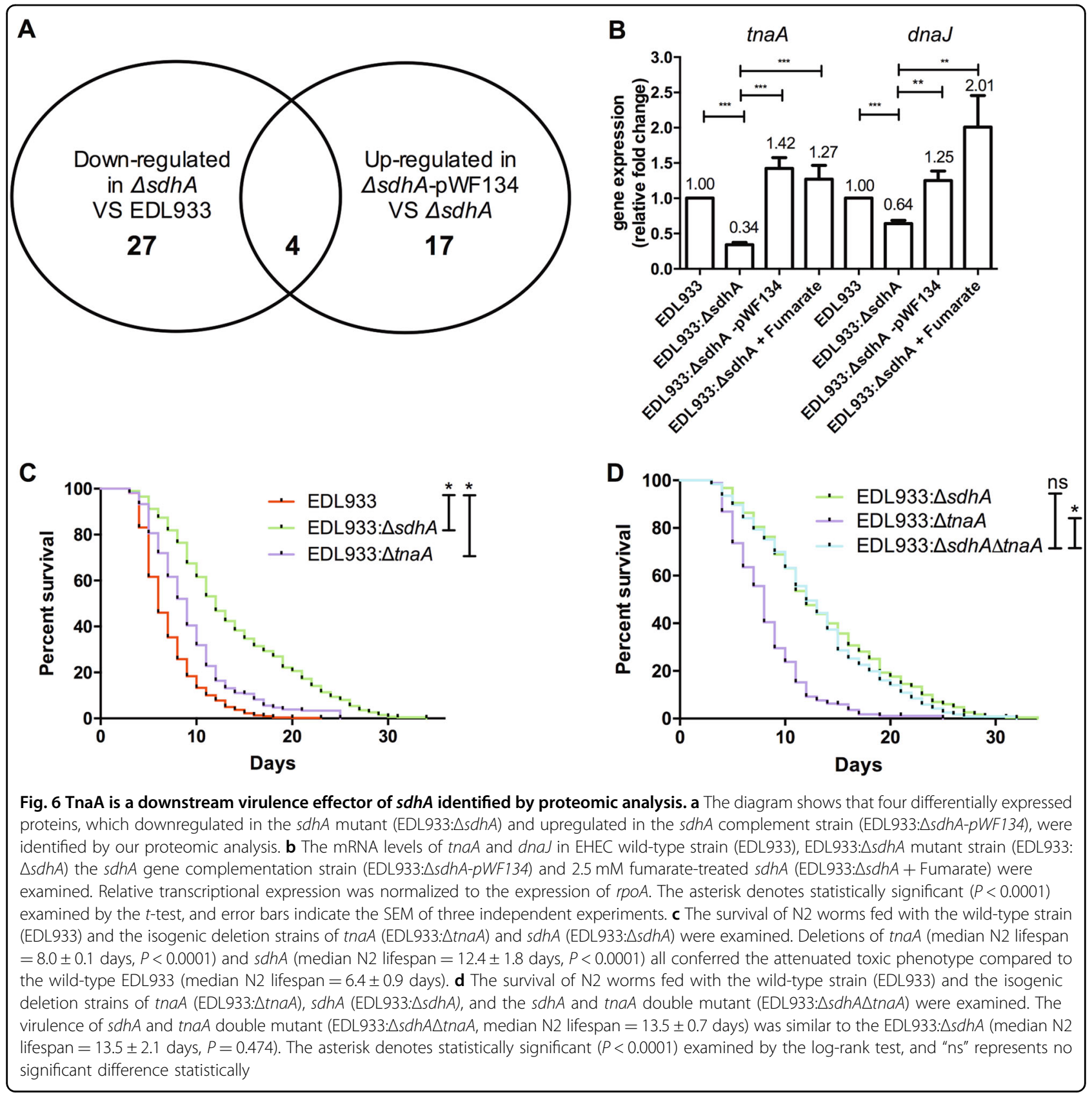

Table 1 Proteins with differential expression in the wild-type EHEC strain (EDL933), the isogenic sdhA deletion mutant

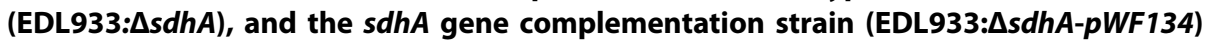

\begin{tabular}{|c|c|c|c|c|c|c|c|}
\hline \multirow[t]{2}{*}{ Protein Name } & \multirow[t]{2}{*}{ Gene name } & \multicolumn{3}{|c|}{$\Delta s d h A / E D L 933$} & \multicolumn{3}{|c|}{$\Delta s d h A-p W F 134 / \Delta s d h A$} \\
\hline & & $P$ value & Fold change & Trend & $P$ value & Fold change & Trend \\
\hline Succinate dehydrogenase flavoprotein subunit & $\operatorname{sdh} A$ & 0.001 & -9.45 & $\downarrow$ & 0.014 & 70.92 & $\uparrow$ \\
\hline Succinate dehydrogenase iron-sulfur subunit & $\operatorname{sdh} B$ & 0.002 & -10.31 & $\downarrow$ & $<0.0001$ & 65.79 & $\uparrow$ \\
\hline Tryptophanase & thaA & 0.010 & -1.77 & $\downarrow$ & 0.008 & 2.27 & $\uparrow$ \\
\hline Molecular chaperone & dnaJ & 0.010 & -100 & $\downarrow$ & $<0.0001$ & 100 & $\uparrow$ \\
\hline
\end{tabular}




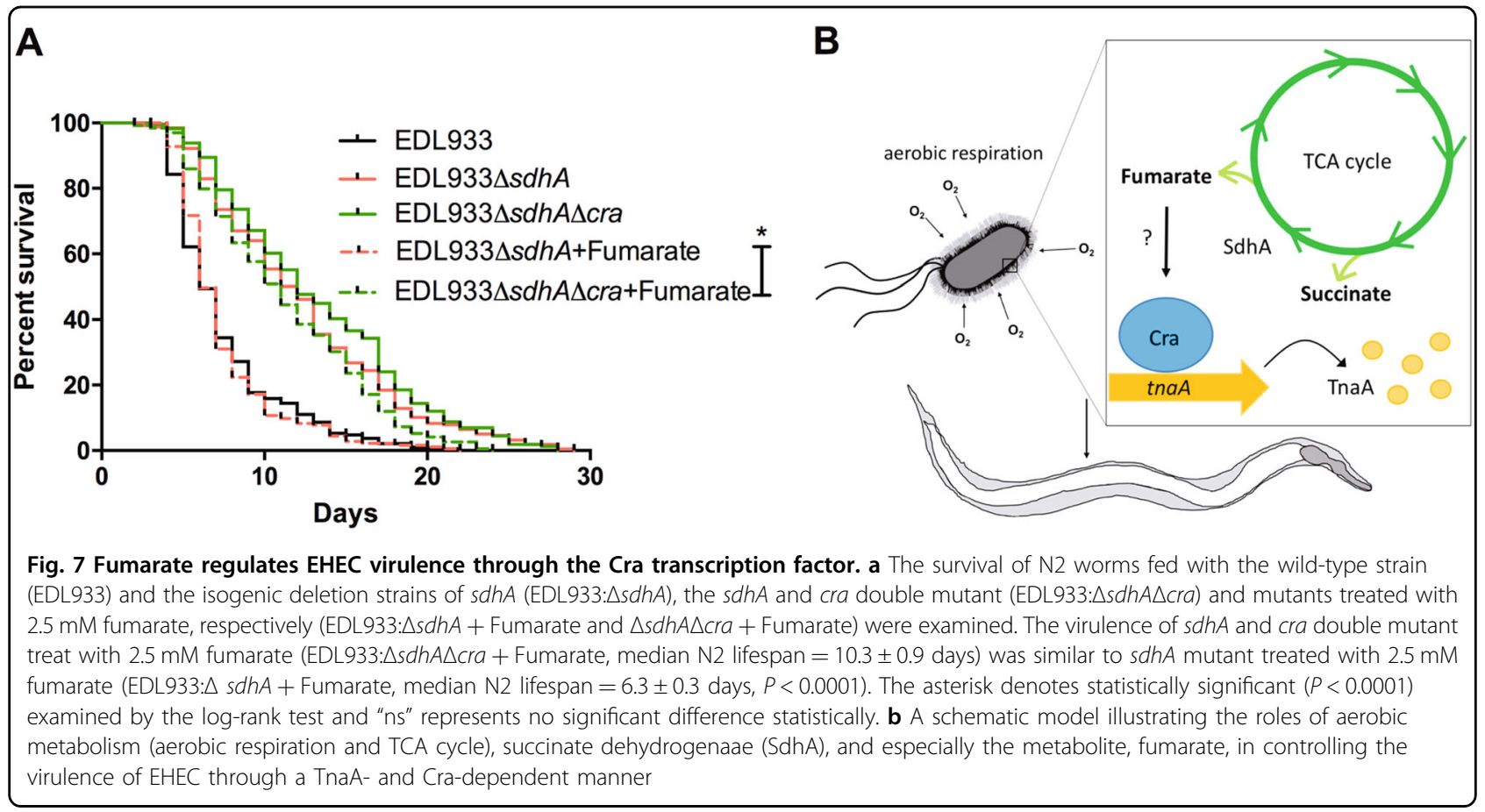

EHEC sdhA gene disruption. Interestingly, the attenuated pathogenicity of the $s d h A$ mutant and the expression of tnaA in the $s d h A$ mutant were significantly restored by supplementing with fumarate, the metabolite of SdhA, in a Cra-dependent manner. A model summarizing our findings is presented in the Fig. 7b. However, whether fumarate can directly or indirectly modulate Cra and how Cra can regulate the transcription of TnaA are warranted to test. Nevertheless, our results demonstrated that the bacterial metabolite fumarate played a major role in the pathogenesis of EHEC in vivo.

The TCA cycle has also been reported to be required for the virulence of the other enteric pathogens, Salmonella enterica serovar Typhimurium and uropathogenic $E$. $\mathrm{coli}^{35-38}$. Our results are in agreement with these reports, and we demonstrated, to our knowledge, for first time, the mechanism of the Sdh complex and mode of action of its catabolite, fumarate, in controlling EHEC virulence in vivo. Aforementioned that there is a relatively aerobic zone adjacent to the mucosal surface of the intestinal epithelial cells caused by diffusion of oxygen from the microvilli capillary network ${ }^{7}$. It has been reported that variable oxygen content also modulates EHEC virulence in a vertical diffusion chamber system ${ }^{39}$. In addition, aerobic respiratory metabolism has also been reported to contribute to EHEC colonization of mouse intestine ${ }^{6,40}$. Our results here are in accordance with these observations, we demonstrated that EHEC is more toxic upon aerobiosis and is less toxic upon anaerobiosis to $C$. elegans.
Multiple metabolites of central carbon metabolism have been reported to regulate C. elegans lifespan. For example $\alpha$-ketoglutarate, malate, fumarate, oxaloacetate, and acetate can extend C. elegans lifespan ${ }^{41-44}$. On the other hand, glucose shortens the lifespan of C. elegans ${ }^{45}$. Moreover, some metabolites such as tryptophan, indole, glycogen, and maltose also modulate the virulence of pathogenic bacteria ${ }^{3,4,46}$. In our study, we demonstrated that fumarate supplement in the $s d h A$ mutant could significantly reverse the attenuated toxicity of the $s d h A$ mutant. However, there was no obvious effect on the toxicity when the wild-type E. coli EDL933 strain and OP50 strain were treated with fumarate. A recent report suggested that fumarate increases the lifespan of $C$. ele$\operatorname{gans}^{44}$, while we did not observe the same effect in our studies. This discrepancy may be the result of the different culture conditions of C. elegans and the concentration of fumarate used in our study.

Interestingly, it has been suggested that oxygen levels varying in the gastrointestinal tract modulate intracellular enteric pathogen Shigella flexneri virulence ${ }^{7}$. The fnr gene deletion mutant reduces the Shigella flexneri 1 colonization in the intestine and reduces the destruction of the mucosal surface of villi. However, in our study, the EDL933 mutant with fnr gene deletion expressed similar toxicity as wild-type. These results suggested that the regulation of virulence by the switch between aerobic and anaerobic respirations is strain-specific and may be Fnrindependent in EHEC. Cra is a universal regulator that regulates genes involved in carbon metabolism and can 
also regulate EHEC virulence expression dependent on glucose concentration ${ }^{8}$. A recent report showed that metabolism and oxygen availability can affect EHEC virulence via $\mathrm{Cra}^{47}$. Here we demonstrated that EHEC is less toxic toward C. elegans under anaerobic culture and this regulation might be controlled by the metabolite, fumarate. Consistent with the idea that when EHEC shifts to the aerobic zone of gut epithelial cells in GI tract, EHEC activates T3SS expression enhancing its adherence to epithelial cells and this activation is dependent on Cra regulation ${ }^{39,47}$.

Pathogenic E. coli may require certain metabolites, nutrients or specific metabolic pathways different from commensal $E$. coli and these differences may contribute to the pathogenicity. On the other hand, pathogens may utilize alternative pathways or mechanisms to evade the cell toxic metabolites or metabolic pathways resulting in reducing its pathogenicity. We envision that such EHECspecific metabolite or metabolic pathways could be the potential targets for anti-infection.

\section{Acknowledgements}

We thank Drs. Ching-Hao Teng, Hung-Yu Shu, and the E. coli research group at NCKU for critical comments and helpful discussions. We thank the Caenorhabditis Genetics Center (CGC), which is supported by the National Institutes of Health (United States), Office of Research Infrastructure Programs (P40 OD010440), for the C. elegans strains. We are grateful to the assistance from the Taiwan C. elegans core facility, funded by the Minister of Science and Technology (MOST) Taiwan, and feedback from the Taiwan worm research community. We thank Miranda Loney for editing the manuscript. This work is supported by the Ministry of Science and Technology (MOST) grants (1012311-B-006-005, 102-2311-B-006-005, 102-2321-B-006-022, and 104-2321-B006-019) to CSC and the Next-generation Pathway of Taiwan Precision Medicine Program (TCPMP) grant (MP10604-007) to J.-W.C. The funders had no role in study design, data collection, and analysis, decision to publish, or preparation of the manuscript.

\section{Author details}

'Department of Biochemistry and Molecular Biology, College of Medicine, National Cheng Kung University, Tainan, Taiwan. ${ }^{2}$ Institute of Basic Medical Sciences, College of Medicine, National Cheng Kung University, Tainan, Taiwan. ${ }^{3}$ Department of Clinical Laboratory Sciences and Medical Biotechnology, National Taiwan University, Taipei, Taiwan. ${ }^{4}$ The Graduate Institute of Integrated Medicine, China Medical University Taichung Taiwan. ${ }^{5}$ Graduate Institute of Biochemical Sciences, Technology Commons, Center for Systems Biology, National Taiwan University, Taipei, Taiwan. ${ }^{6}$ Department of Microbiology and Immunology, College of Medicine, National Cheng Kung University, Tainan, Taiwan

\section{Author contributions}

C.-J.K., S.-T.W., J.-W.C., and .C-M.L., C.-S.C. conceived and designed the experiments. C.-J.K., S.-T.W., C.-M.L., J.-W.C., C.-R.H., D.-Y.L., and T.-C.C. performed the experiments. C.-J.K., S.-T.W., C.-M.L., H.-C.C., D.-Y.L., G.-D.C., T.-C.C., J.W.C., and C.-S.C. analyzed the data. H.-C.C. and G.-D.C. contributed reagents/ materials/analysis tools. C.-J.K., C.-M.L., J.-W.C., and C.-S.C. wrote the paper.

\section{Conflict of interest}

The authors declare that they have no conflict of interest.

\section{Publisher's note}

Springer Nature remains neutral with regard to jurisdictional claims in published maps and institutional affiliations.
Supplementary Information accompanies this paper at https://doi.org/ 10.1038/s41419-018-0423-2.

Received: 30 November 2017 Revised: 13 February 2018 Accepted: 16 February 2018

Published online: 07 March 2018

\section{References}

1. Nguyen, Y. \& Sperandio, V. Enterohemorrhagic E. coli (EHEC) pathogenesis. Front. Cell. Infect. Microbiol. 2, 90 (2012).

2. Lim, J. Y., Yoon, J. W. \& Hovde, C. J. A brief overview of Escherichia coli O157:H7 and its plasmid O157. J. Microbiol. Biotechnol. 20, 5-14 (2010).

3. Anyanful, A. et al. Paralysis and killing of Caenorhabditis elegans by enteropathogenic Escherichia coli requires the bacterial tryptophanase gene. Mol. Microbiol. 57, 988-1007 (2005).

4. Bommarius, B. et al. A family of indoles regulate virulence and Shiga toxin production in pathogenic E. coli. PLOS ONE. 8, e54456 (2013).

5. Hirakawa, H., Kodama, T., Takumi-Kobayashi, A., Honda, T. \& Yamaguchi, A. Secreted indole serves as a signal for expression of type III secretion system translocators in enterohaemorrhagic Escherichia coli O157:H7. Microbiology 155(Pt 2), 541-550 (2009).

6. Jones, S. A. et al. Respiration of Escherichia coli in the mouse intestine. Infect. Immun. 75, 4891-4899 (2007).

7. Marteyn, B. et al. Modulation of Shigella virulence in response to available oxygen in vivo. Nature 465, 355-358 (2010).

8. Njoroge, J. W., Nguyen, Y., Curtis, M. M., Moreira, C. G. \& Sperandio, V. Virulence meets metabolism: Cra and $\mathrm{KdpE}$ gene regulation in enterohemorrhagic Escherichia coli. mBio 3, e00280-12 (2012).

9. Hernandez-Doria, J. D. \& Sperandio, V. Nutrient and chemical sensing by intestinal pathogens. Microbes Infect. 15, 759-764 (2013).

10. Rohmer, L., Hocquet, D. \& Miller, S. I. Are pathogenic bacteria just looking for food? Metabolism and microbial pathogenesis. Trends Microbiol. 19, 341-348 (2011).

11. Barnett Foster, D. Modulation of the enterohemorrhagic E. coli virulence program through the human gastrointestinal tract. Virulence 4, 315-323 (2013).

12. Keeney, K. M. \& Finlay, B. B. Enteric pathogen exploitation of the microbiotagenerated nutrient environment of the gut. Curr. Opin. Microbiol. 14, 92-98 (2011).

13. Tobe, T., Nakanishi, N. \& Sugimoto, N. Activation of motility by sensing shortchain fatty acids via two steps in a flagellar gene regulatory cascade in enterohemorrhagic Escherichia coli. Infect. Immun. 79, 1016-1024 (2011).

14. Zumbrun, S. D. et al. Dietary choice affects Shiga toxin-producing Escherichia coli (STEC) 0157:H7 colonization and disease. Proc. Natl Acad. Sci. USA 110, E2126-E2133 (2013).

15. Kendall, M. M., Gruber, C. C., Parker, C. T. \& Sperandio, V. Ethanolamine controls expression of genes encoding components involved in interkingdom signaling and virulence in enterohemorrhagic Escherichia coli O157:H7. mBio 3, pii: e00050-12 (2012).

16. Gonyar, L. A. \& Kendall, M. M. Ethanolamine and choline promote expression of putative and characterized fimbriae in enterohemorrhagic Escherichia coli O157:H7. Infect. Immun. 82, 193-201 (2014).

17. Pacheco, A. R. et al. Fucose sensing regulates bacterial intestinal colonization. Nature 492, 113-117 (2012).

18. Mohawk, K. L. \& O'Brien, A. D. Mouse models of Escherichia coli O157:H7 infection and shiga toxin injection. J. Biomed. Biotechnol. 2011, 258185 (2011).

19. Chou, T. C. et al. Enterohaemorrhagic Escherichia coli O157:H7 Shiga-like toxin 1 is required for full pathogenicity and activation of the p38 mitogen-activated protein kinase pathway in Caenorhabditis elegans. Cell. Microbiol. 15, 82-97 (2013).

20. Brenner, S. The genetics of Caenorhabditis elegans. Genetics 77, 71-94 (1974).

21. Datsenko, K. A. \& Wanner, B. L. One-step inactivation of chromosomal genes in Escherichia coli K-12 using PCR products. Proc. Natl Acad. Sci. USA 97, 6640-6645 (2000).

22. Muniesa, M., Serra-Moreno, R., Acosta, S., Hernalsteens, J. P. \& Jofre, J. Use of the lambda Red recombinase system to produce recombinant prophages carrying antibiotic resistance genes. BMC Mol. Biol. 7, 31 (2006). 
23. Valdivia, R. H. \& Falkow, S. Bacterial genetics by flow cytometry: rapid isolation of Salmonella typhimurium acid-inducible promoters by differential fluorescence induction. Mol. Microbiol. 22, 367-378 (1996).

24. Kuo, C. J. et al. Mutation of the enterohemorrhagic Escherichia coli core LPS biosynthesis enzyme RfaD confers hypersusceptibility to host intestinal innate immunity in vivo. Front. Cell. Infect. Microbiol. 6, 82 (2016).

25. Shevchenko, A., Tomas, H., Havlis, J., Olsen, J. V. \& Mann, M. In-gel digestion for mass spectrometric characterization of proteins and proteomes. Nat. Protoc. 1, 2856-2860 (2006)

26. Feinbaum, R. L. et al. Genome-wide identification of Pseudomonas aeruginosa virulence-related genes using a Caenorhabditis elegans infection model. PLoS Pathog. 8, e1002813 (2012).

27. Simonsen, K. T. et al. A role for the RNA chaperone $\mathrm{Hfq}$ in controlling adherent-invasive Escherichia coli colonization and virulence. PLOS ONE 6, e16387 (2011)

28. Bojer, M. S., Jakobsen, H., Struve, C., Krogfelt, K. A. \& Lobner-Olesen, A. Lack of the RNA chaperone $\mathrm{Hfq}$ attenuates pathogenicity of several Escherichia coli pathotypes towards Caenorhabditis elegans. Microbes Infect. 14, 1034-1039 (2012).

29. Cecchini, G., Schroder, I., Gunsalus, R. P. \& Maklashina, E. Succinate dehydrogenase and fumarate reductase from Escherichia coli. Biochim. Biophys. Acta 1553, 140-157 (2002).

30. Unden, G. \& Bongaerts, J. Alternative respiratory pathways of Escherichia coli: energetics and transcriptional regulation in response to electron acceptors. Biochim. Biophys. Acta Bioenerg. 1320, 217-234 (1997).

31. Sell, S. M., Eisen, C., Ang, D., Zylicz, M. \& Georgopoulos, C. Isolation and characterization of dnaJ null mutants of Escherichia coli. J. Bacteriol. 172, 4827-4835 (1990)

32. Unden, G., Strecker, A., Kleefeld, A., Kim, O. B. C4-dicarboxylate utilization in aerobic and anaerobic growth. EcoSal Plus. 7 (2016) doi: 10.1128/ecosalplus. ESP-0021-2015.

33. Njoroge, J. W., Gruber, C. \& Sperandio, V. The interacting Cra and KdpE regulators are involved in the expression of multiple virulence factors in enterohemorrhagic Escherichia coli. J. Bacteriol. 195, 2499-2508 (2013).

34. Kochanowski, K. et al. Functioning of a metabolic flux sensor in Escherichia coli. Proc. Natl Acad. Sci. USA 110, 1130-1135 (2013).
35. Bowden, S. D., Ramachandran, V. K., Knudsen, G. M., Hinton, J. C. \& Thompson, A. An incomplete TCA cycle increases survival of Salmonella Typhimurium during infection of resting and activated murine macrophages. PLOS ONE $\mathbf{5}$, e13871 (2010)

36. Mercado-Lubo, R., Gauger, E. J., Leatham, M. P., Conway, T. \& Cohen, P. S. A Salmonella enterica serovar typhimurium succinate dehydrogenase/fumarate reductase double mutant is avirulent and immunogenic in BALB/C mice. Infect. Immun. 76, 1128-1134 (2008).

37. Alteri, C. J., Smith, S. N. \& Mobley, H. L. Fitness of Escherichia coli during urinan tract infection requires gluconeogenesis and the TCA cycle. PLoS Pathog. $\mathbf{5}$ e1000448 (2009)

38. Tchawa Yimga, M. et al. Role of gluconeogenesis and the tricarboxylic acid cycle in the virulence of Salmonella enterica Serovar Typhimurium in BALB/C mice. Infect. Immun. 74, 1130-1140 (2006).

39. Schuller, S. \& Phillips, A. D. Microaerobic conditions enhance type III secretion and adherence of enterohaemorrhagic Escherichia coli to polarized human intestinal epithelial cells. Environ. Microbiol. 12, 2426-2435 (2010).

40. Jones, S. A. et al. Anaerobic respiration of Escherichia coli in the mouse intestine. Infect. Immun. 79, 4218-4226 (2011).

41. Mouchiroud, L. et al. Pyruvate imbalance mediates metabolic reprogramming and mimics lifespan extension by dietaryrestriction in Caenorhabditis elegans. Aging Cell. 10, 39-54 (2011).

42. Chin, R. M. et al. The metabolite alpha-ketoglutarate extends lifespan by inhibiting ATP synthase and TOR. Nature 510, 397-401 (2014).

43. Chuang, M. H., Chiou, S. H., Huang, C. H., Yang, W. B. \& Wong, C. H. The lifespan-promoting effect of acetic acid and Reishi polysaccharide. Bioorg. Med. Chem. 17, 7831-7840 (2009).

44. Edwards, C. B., Copes, N., Brito, A. G., Canfield, J. \& Bradshaw, P. C. Malate and fumarate extend lifespan in Caenorhabditis elegans. PLoS ONE 8, e58345 (2013).

45. Lee, S. J., Murphy, C. T. \& Kenyon, C. Glucose shortens the life span of C. elegans by downregulating DAF-16/FOXO activity and aquaporin gene expression. Cell. Metab. 10, 379-391 (2009).

46. Jones, S. A. et al. Glycogen and maltose utilization by Escherichia coli O157:H7 in the mouse intestine. Infect. Immun. 76, 2531-2540 (2008).

47. Carlson-Banning, K. M. \& Sperandio, V. Catabolite and oxygen regulation of enterohemorrhagic Escherichia coli virulence. mBio 7, pii: e01852-16 (2016). 